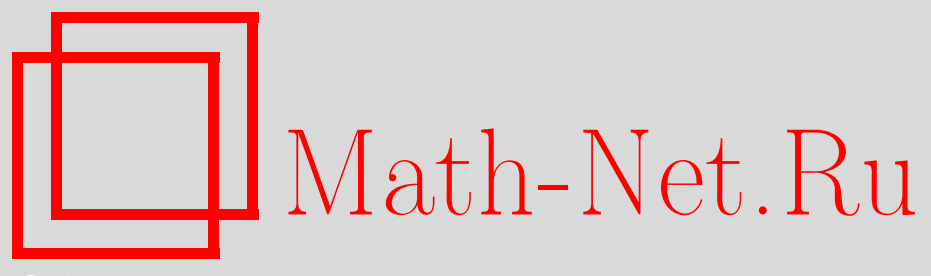

Д. В. Аносов, В. П. Лексин, О работах Андрея Андреевича Болибруха по аналитической теории дифференциальных уравнений, УМН, 2011, том 66, выпуск 1, 3-36

DOI: https://doi.org/10.4213/rm9401

Использование Общероссийского математического портала Math-Net.Ru подразумевает, что вы прочитали и согласны с пользовательским соглашением http://www . mathnet.ru/rus/agreement

Параметры загрузки:

IP : 52.6 .47 .48

26 апреля 2023 г., $17: 12: 40$

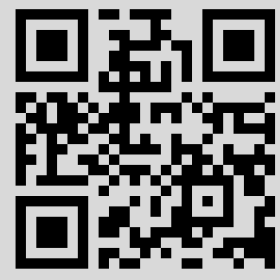




\title{
О работах Андрея Андреевича Болибруха по аналитической теории дифференциальных уравнений
}

\author{
Д. В. Аносов, В. П. Лексин
}

В статье дан обзор работ А. А. Болибруха по новым направлениям исследований в аналитической теории дифференциальных уравнений, которые возникли вследствие его сенсационного контрпримера к проблеме Римана-Гильберта. Также приводится обзор работ его прямых учеников, в которых развиваются темы, начатые в работах Болибруха. В данном обзоре мы в основном сосредоточиваем внимание на роли приводимости и неприводимости систем линейных дифференциальных уравнений и их представлений монодромии. Дано краткое описание результатов по многомерной проблеме Римана-Гильберта и изомонодромным деформациям фуксовых систем, а также кратко описаны основные методы современной аналитической теории дифференциальных уравнений.

Библиография: 69 названий.

Ключевые слова: регулярные и фуксовы системы линейных дифференциальных уравнений, представления монодромии мероморфных систем дифференциальных уравнений, проблема Римана-Гильберта, приводимые и неприводимые представления монодромии и системы дифференциальных уравнений, изомонодромные деформации.

\section{СОДЕРЖАНИЕ}

1. Начало исследований: теория многомерных регулярных и фуксовых систем........................................

2. Регулярные и фуксовы системы на сфере Римана. Контрпримеры к про-

блеме Римана-Гильберта ................................

3. Приводимость и неприводимость представлений и систем дифференци-

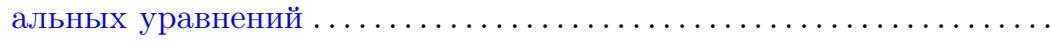

4. Фуксовы уравнения и фуксовы системы. Иррегулярные системы. Обобщенная проблема Римана-Гильберта .....................

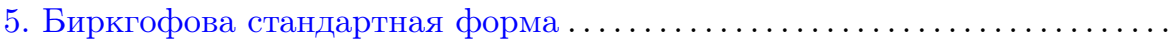

6. Изомонодромные деформации мероморфных систем. Изомонодромное

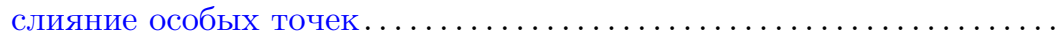

Работа выполнена при поддержке программы "Ведущие научные школы" (грант НШ-8508.2010.1). 
7. Методы решения задач аналитической теории дифференциальных

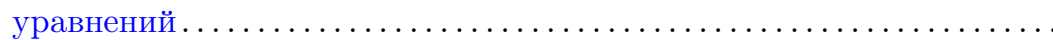

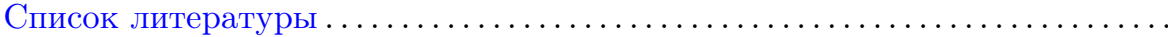

30 января 2010 г. исполнилось 60 лет со дня рождения выдающегося российского математика Андрея Андреевича Болибруха. Подробно о жизни и творчестве Андрея Андреевича можно прочитать в статье [1] (см. также дополнение в книге [2]). Мы здесь только кратко напомним некоторые факты его биографии, а затем перейдем к обзору его работ и работ учеников, продолживших его исследования. Он родился в Москве в семье военнослужащего. Его отец, Андрей Власьевич Болибрух, в конце своей службы, прерванной преждевременной смертью, имел звание генерал-лейтенанта и занимал должность зам. командующего Прибалтийского военного округа. Мама, Татьяна Ивановна, всецело посвятила себя воспитанию детей. Андрей Андреевич не пошел по стопам отца, а связал свою жизнь с наукой. Он стал математиком, чему в немалой степени способствовали ранние успехи в математических олимпиадах и стройная система работы с талантливой молодежью, которая успешно действовала тогда в стране, отметим также "дух времени", который побуждал молодежь мечтать о научной карьере. К сожалению, судьбе было угодно распорядиться так, что тяжелая болезнь рано оборвала жизнь Андрея Андреевича. Он умер примерно в том же возрасте, что и его отец. Он, так же как его отец, добился признания своего труда, будучи академиком и занимая высокие посты в Российской академии наук.

В статье [1], указанной выше, речь, в основном, шла о главном достижении Андрея Андреевича: построении контрпримера к решению классической проблемы Римана-Гильберта, которая к тому времени примерно 80 лет считалась решенной в положительном смысле. Мы также указывали в [1], что этот результат Андрея Андреевича естественно вел к возникновению новых направлений исследований в аналитической теории дифференциальных уравнений. Здесь мы хотим более подробно рассказать о том, что было сделано самим Андреем Андреевичем, а также его прямыми учениками в новых направлениях аналитической теории дифференциальных уравнений. Мы сделаем акцент на результатах, полученных в классической ситуации, т. е. для аналитических дифференциальных уравнений на сфере Римана. Точнее, основная часть обзора будет посвящена циклу результатов, связанных со структурой приводимости (и, в частности, неприводимости) систем уравнений и их представлений монодромии. Что касается результатов о дифференциальных уравнениях на компактных римановых поверхностях рода больше нуля и на многомерных комплексных многообразиях, то мы скажем о них очень сжато, мотивируя тем, что их можно найти в обзорах [3], [4], опубликованных в УМН. Сохраняя хронологию творческого пути А. А., мы хотим вначале рассказать о первых работах Андрея Андреевича по аналитической теории дифференциальных уравнений на многомерных комплексных многообразиях, которые сыграли существенную роль в понимании сути многих проблем классической аналитической теории дифференциальных уравнений. В статье [1] и конце книги [2] имеется полный список научных работ А.А. Болибруха, тем не менее, для удобства читателей, мы в списке литературы приводим полные ссылки на основные работы 
А. А. Болибруха. Далее для краткости мы будем пользоваться аббревиатурой А. А. при упоминании А. А. Болибруха.

\section{1. Начало исследований: теория многомерных регулярных и фуксовых систем}

Исследования А. А. по аналитической теории дифференциальных уравнений начались с изучения регулярных и фуксовых линейных интегрируемых мероморфных пфаффовых систем на многомерных комплексных многообразиях. Точнее, изучался локальный вид фундаментальных матриц решений регулярных и фуксовых систем и критерии фуксовости для регулярных систем. Достаточно подробный обзор результатов, полученных А. А. по многомерной теории регулярных и фуксовых систем, имеется в обзоре [4], опубликованном в УМН, и потому мы не будем здесь давать точных формулировок этих результатов, ограничив себя передачей их смысла и указанием их места в теории, напомнив при этом основные определения, необходимые для понимания текста.

По определению, регулярная система - это интегрируемая мероморфная линейная система Пфаффа

$$
d y=\Omega y
$$

на $\mathbb{C}^{m}$, все решения которой имеют степенной рост в точках полярного множества особенностей $A=A_{1} \cup \cdots \cup A_{n} \subset \mathbb{C}^{m}$ формы $\Omega$ системы.

Здесь $\Omega$ - матричная мероморфная дифференциальная 1-форма с полярным множеством особенностей $A$ голоморфная вне $A$. Мы предполагаем, что $A$ удовлетворяет условию общего положения, т. е. является объединением неособых подмногообразий $A_{j}, j=1, \ldots, n$, трансверсально пересекающихся в $\mathbb{C}^{m}$. Интегрируемость системы (1) означает ее интегрируемость в смысле Фробениуса, которая в случае линейных систем Пфаффа равносильна равенству $d \Omega=\Omega \wedge \Omega$. Пусть размеры матрицы $\Omega$ будут $p \times p$ и значения вектор-функции $y(z)$ в $\mathbb{C}^{p}$ записываются столбцами из $p$ строк. Под степенным ростом $y(z)$ в точке $a \in A$ мы понимаем как настоящий степенной рост вектор-функции $y(z)$, так и ее степенное убывание. В определении степенного роста решения $y(z)$ используются стандартные эрмитовы формы на $\mathbb{C}^{p}$ и $\mathbb{C}^{m}$ и отвечающие им нормы. Степенной рост (или убывание) решения $y(z)$ системы (1) в точке $a \in A$ означает, что относительно стандартных норм в $\mathbb{C}^{p}$ и в $\mathbb{C}^{m}$ для некоторой положительной вещественной константы $C$ и некоторого целого числа $N$ имеется степенная оценка $|y(z)| \leqslant C|z-a|^{-N}$ роста вектор-функции $y(z)$ в точке $a \in A$ при приближении к ней по любому достаточно малому и узкому открытому сектору $U \subset \mathbb{C}^{m} \backslash A$ с вершиной в $a \in A$. Точнее, рост мы рассматриваем в поднятии указанного сектора в универсальное накрытие (при этом мы не будем различать в обозначениях точки и их координаты в секторе $U$ и в его поднятии в универсальное накрытие) дополнения $\mathbb{C}^{m} \backslash A$. Сектор $U \subset \mathbb{C}^{m} \backslash A$ с вершиной в $a \in A$ считается достаточно узким, если его проекция на любое одномерное координатное пространство $\mathbb{C}_{i}^{1}$ является сектором конечного раствора с вершинами в точке $a_{i} \in \mathbb{C}_{i}^{1}\left(a_{i}-i\right.$-я координата точки $\left.a \in A\right)$, не содержащим всей комплексной плоскости $\mathbb{C}_{i}^{1}$.

Система (1) называется регулярной в точках ее особого множества $A$, если она регулярна в каждой точке множества $A$. 
Система $d y=\Omega y$ с особенностями на $A$ называется фуксовой системой в точке $a \in A$, если 1-форма $\Omega$ системы имеет полюсы первого порядка (в многомерном случае $m>1$ еще требуется, чтобы они были логарифмического типа) на $A$ в окрестности точки $a$ и удовлетворяет условию интегрируемости $d \Omega=\Omega \wedge \Omega$.

По определению, мероморфная 1-форма $\Omega$ имеет полюсы логарифмического muna на $A$ в точке $a \in A$, если в достаточно малой окрестности $V$ точки $a \in A$ форма $\Omega$ системы имеет вид

$$
\Omega=\sum_{i=1}^{k} B_{i}(z) \frac{d f_{i}(z)}{f_{i}(z)}+\Omega_{0},
$$

где $f_{i}(z), i=1, \ldots, k,-$ голоморфные функции, нули которых задают компоненты $A_{i}$ особого множества $A$, проходящие через точку $a \in A$, матричные функции $B_{i}(z), i=1, \ldots, k,-$ голоморфные функции в окрестности $V$ и $B_{i}(a) \neq 0$ для $i=1, \ldots, k$, а дифференциальная 1-форма $\Omega_{0}$ - голоморфна в окрестности $V$.

По определению, мероморфная система $d y=\Omega y-$ фуксова на $\mathbb{C}^{m}$, если она фуксова в каждой точке $a$ своего множества особенностей $A$.

Сразу отметим, что А.А. доказал следующий факт: любая фуксова система на $\mathbb{C}^{m}$ с множеством особенностей общего положения является регулярной системой.

Основной целью первых работ [5]-[8] было обобщение на интегрируемые многомерные $(m>1)$ мероморфные регулярные пфаффовы системы результатов А. Левеля [9] (формулировки можно найти в следующем разделе) по классификации асимптотик фундаментальных матриц решений фуксовых и регулярных систем на сфере Римана (т.е. на существенно одномерном комплексном многообразии) и отыскание локальных и глобальных критериев фуксовости регулярных систем в многомерном случае, в обобщение аналогичных результатов Левеля в одномерном случае. Результаты Левеля позволяли обобщить геометрические методы теории голоморфных векторных расслоений, использованные Х. Рёрлем [10] в 1957 г. в аналитической теории дифференциальных уравнений для решения классической проблемы Римана-Гильберта. Ю. И. Манин [11] усовершенствовал технику Рёрля в другом, алгебраическом, направлении, рассматривая вместо голоморфных расслоений со связностью алгебраические D-модули. П. Делинь [12] в 1970 г. расширил технику Рёрля, рассматривая продолжения расслоений вместе с фуксовыми (логарифмическими) связностями (у таких связностей форма связности локально является формой с полюсами первого порядка логарифмического типа, как объяснено выше), причем он определил такие продолжения и на многомерных комплексных многообразиях, назвав получаемые продолжения "каноническими продолжениями". Он также дал геометрическую интерпретацию понятий, введенных Маниным. Делинь сформулировал свой вариант проблемы Римана-Гильберта, решение которого состояло в построение продолжения расслоения с интегрируемой связностью, определенного на дополнении к множеству особенностей, до расслоения с интегрируемой фуксовой связностью на всем многообразии. Этот вариант проблемы Римана-Гильберта можно рассматривать как на одномерных, так и на многомерных комплексных многообразиях, но он существенно слабее классического варианта проблемы Римана-Гильберта для класса фуксовых систем, 
на что указывал Н. Катц [13]. Такой вариант проблемы Римана-Гильберта всегда имеет положительное решение, как в одномерном случае, так и в многомерном случае, когда фиксируемое множество особенностей искомой фуксовой (или, в другой терминологии, логарифмической) связности есть объединение неособых подмногообразий коразмерности один, имеющих только трансверсальные пересечения. Технику продолжений расслоений со связностью можно сделать существенно более удобной и наглядной (в применении к проблеме Римана-Гильберта), если использовать язык главных расслоений и скольжений в универсальном накрытии, на что постоянно указывал Д. В. Аносов [14]-[16].

Однако сам Левель не связывал свои исследования с подходами Рёрля и Делиня к проблеме Римана-Гильберта и не рассматривал новые продолжения голоморфных расслоений со связностью, существование которых (а также их отличие от канонических продолжений Делиня-Рёрля) вытекало из его результатов. Следуя Делиню, в многомерном случае новые продолжения "по Левелю", отличные от канонических, можно было построить только при условии обобщения результатов Левеля о локальных асимптотиках регулярных и фуксовых систем на многомерный случай. Р. Жерар, ученик Левеля, сделал такое обобщение, и А.А. под влиянием его работы занялся этой задачей, пытаясь разобраться с некоторыми нестыковками результатов Жерара о структуре фундаментальной матрицы решений многомерных регулярных и фуксовых систем с результатами в одномерной ситуации, описанными в работах Левеля и, еще раньше, для фуксовых систем, в известной книге Гантмахера "Теория матриц" [17]. Первоначально предполагалось, что некоторые различия связаны с эффектами многомерности, однако вскоре эти предположения отпали и была обнаружена неточность в определении нормирований (это и приводило к “дополнительным свойствам" фундаментальных матриц решений) аналитических функций степенного роста на многомерных комплексных многообразиях. А. А. дал правильное определение нормирований и пришел к правильному описанию фундаментальных матриц решений многомерных фуксовых систем, а также сформулировал аналоги локального и глобального критериев фуксовости Левеля для многомерных регулярных систем. Позже в работе [18] теория соотношений Фукса (равенства и неравенства Фукса) для уравнений и систем на сфере Римана была обобщена на расслоения со связностями на многомерных компактных кэлеровых многообразиях. Отметим также, что теория многомерных регулярных пфаффовых систем позволяет геометрически наглядно сформулировать понятие изомонодромных деформаций фуксовых систем на сфере Римана и классифицировать возможные типы таких изомонодромных деформаций. Об этом пойдет речь в конце статьи.

\section{2. Регулярные и фуксовы системы на сфере Римана. Контрпримеры к проблеме Римана-Гильберта}

Напомним основные понятия (см. [2], [16], [19]), которые необходимы для формулировки классической проблемы Римана-Гильберта в классе фуксовых систем, и сформулируем эту проблему, а также ее ослабленный вариант. 
Рассмотрим линейную мероморфную систему на сфере Римана $\overline{\mathbb{C}}=\mathbb{C} \cup$ $\{z=\infty\}:$

$$
\frac{\mathrm{d} y(z)}{\mathrm{d} z}=\left(\sum_{i=1}^{n} \frac{B_{i}}{z-a_{i}}\right) y(z)
$$

Здесь

$$
y(z)=\left(\begin{array}{c}
y_{1}(z) \\
\vdots \\
y_{p}(z)
\end{array}\right)
$$

- неизвестная аналитическая вектор-функция, а коэффициенты $B_{i}, i=1, \ldots, n$, являются постоянными матрицами размера $p \times p$ и удовлетворяют условию

$$
\sum_{i=1}^{n} B_{i}=0
$$

Равенство (3) обеспечивает отсутствие у системы (2) особенности в бесконечно удаленной точке $z=\infty$ сферы Римана $\overline{\mathbb{C}}$, так что система $(2)$ имеет ровно $n$ особых точек из множества $A=\left\{a_{1}, \ldots, a_{n}\right\}$, которое содержится в конечной части сферы Римана. Если среди точек $A$ есть точка $a_{i}=\infty$ и у системы $(2)$ нет других особых точек, то условие (3) также выполняется, т. е. $B_{i}=-\sum_{j=1, j \neq i}^{n} B_{j}$, а систему (2) можно записать (изменив нумерацию особых точек) в виде

$$
\frac{\mathrm{d} y(z)}{\mathrm{d} z}=\left(\sum_{i=1}^{n-1} \frac{B_{i}^{\prime}}{z-a_{i}}\right) y(z)
$$

не требуя равенства $\sum_{i=1}^{n-1} B_{i}^{\prime}=0$.

Системы уравнений $(2)$ или $\left(2^{\prime}\right)$ на сфере Римана называют фуксовыми системами с множеством особенностей $A$.

Выберем для системы (2) неособую точку $z_{0}$ в дополнении $X=\overline{\mathbb{C}} \backslash A \subset \overline{\mathbb{C}}$ на сфере Римана и фундаментальную матрицу голоморфных решений $Y(z)$ системы (2). Столбцами этой матрицы являются элементы некоторого базиса пространства решений системы (2) в достаточно малой окрестности точки $z_{0}$. Рассмотрим произвольный непрерывный путь $\gamma$ в $X$ с началом и концом в точке $z_{0}$, т. е. петлю. Как хорошо известно, при аналитическом продолжении фундаментальной матрицы решений $Y(z)$ вдоль $\gamma$ получим другую фундаментальную матрицу решений $\widetilde{Y}(z)$, которая отличается от исходной фундаментальной матрицы решений на постоянную обратимую матрицу $G_{\gamma}$, а именно, выполняется равенство

$$
Y(z)=\tilde{Y}(z) G_{\gamma}
$$

Матрица $G_{\gamma}$ мультипликативно зависит от петли $\gamma$, а точнее, зависит лишь от гомотопического класса петли $\gamma$, и потому сопоставление

$$
\gamma \mapsto G_{\gamma}
$$


задает некоторое представление

$$
\chi_{m}: \pi_{1}\left(X, z_{0}\right) \rightarrow \mathrm{GL}(p, \mathbb{C})
$$

фундаментальной группы $\pi_{1}\left(X, z_{0}\right)$ в полную линейную группу $\operatorname{GL}(p, \mathbb{C})$. Представление $\chi_{m}$ называется представлением монодромии системы (2) относительно фундаментальной матрицы $Y(z)$. Напомним, что два таких представления $\chi_{1}$ и $\chi_{2}$ называются эквивалентными, если существует постоянная матрица $C \in \mathrm{GL}(p, \mathbb{C})$, для которой выполняются равенства $\chi_{1}(\gamma)=C^{-1} \chi_{2}(\gamma) C$ для любого элемента $\gamma \in \pi_{1}\left(X, z_{0}\right)$. Другой выбор фундаментальной матрицы $Y^{\prime}(z)=Y(z) C$ системы $(2)$ в окрестности точки $z_{0}$ приводит к эквивалентному представлению, таким образом, представление монодромии определено системой (2) неоднозначно, а лишь с точностью до эквивалентности. Часто класс эквивалентности представления $\chi_{m}$, определенного по системе $(2)$, называют представлением монодромии, но в дальнейшем мы будем в основном пользоваться представлением монодромии в первом смысле и будем специально оговаривать те ситуации, когда речь идет о классах эквивалентности представлений. Пусть задано представление монодромии $\chi_{m}$ системы $(2)$ относительно фундаментальной матрицы $Y(z)$. Дополнительно к выбору фундаментальной матрицы $Y(z)$ выберем систему образующих фундаментальной группы $\pi_{1}\left(X, z_{0}\right)=\pi_{1}\left(\overline{\mathbb{C}} \backslash\left\{a_{1}, \ldots, a_{n}\right\}, z_{0}\right)$, представленную "малыми петлями" $\gamma_{j}$, $j=1, \ldots, n$, с началом в $z_{0}$, обходящими соответственно точки $a_{j}, j=1, \ldots, n$, по границам малых кругов с центрами в этих точках и удовлетворяющими соотношению $\gamma_{1} \cdots \gamma_{n}=1$ в группе $\pi_{1}\left(X, z_{0}\right)$. Значения представления $G_{j}=\chi\left(\gamma_{j}\right)$, $j=1, \ldots, n$, на этих образующих называются матрицами монодромии в точках $a_{j}, j=1, \ldots, n$, системы (2) относительно фундаментальной матрицы решений $Y(z)$ или просто матрицами монодромии системы (2). В зависимости от выбора фундаментальной матрицы решений $Y(z)$ матрицы монодромии определены с точностью до общего сопряжения на постоянную матрицу. Сформулируем теорему Левеля из [9], которая составляет основу локальной теории фуксовых систем, изучающей асимптотики решений в окрестности особой точки системы. Сначала выберем такую фундаментальную матрицу решений $Y(z)$ в окрестности точки $z_{0}$, которая при аналитическом продолжении вдоль петли $\gamma_{j}$ имеет верхнетреугольную матрицу монодромии $G_{j}$. Пусть $g_{j}^{k k}-$ элементы на диагонали матрицы $G_{j}$, выберем логарифмы этих чисел $\rho_{j}^{k}=(2 \pi i)^{-1} \log g_{j}^{k k}$, так чтобы вещественная часть $\rho_{j}^{k}$ была заключена между нулем и единицей, $0 \leqslant \operatorname{Re} \rho_{j}^{k}<1$. Возьмем ветвь логарифма $E_{j}=(2 \pi i)^{-1} \log G_{j}$, у которой упорядоченный набор чисел $\left(\rho_{j}^{1}, \ldots, \rho_{j}^{p}\right)$ образует диагональ матрицы $E_{j}$. Утверждается, что для каждой точки $a_{j} \in\left\{a_{1}, \ldots, a_{n}\right\}$ можно выбрать такую фундаментальную матрицу $Y_{j}(z)$ в окрестности $z_{0}$, аналитическое продолжение которой в малую окрестность точки $a_{j}$ имеет в этой окрестности следующий вид:

$$
Y_{j}(z)=U_{j}(z)\left(z-a_{j}\right)^{\Lambda_{j}}\left(z-a_{j}\right)^{E_{j}},
$$

где $U_{j}(z)$ - голоморфно обратимая матрица в этой окрестности, а $\Lambda_{j}=$ $\operatorname{diag}\left(\lambda_{j}^{1}, \ldots, \lambda_{j}^{p}\right)$ - целочисленная диагональная матрица с невозрастающими элементами $\lambda_{j}^{1} \geqslant \cdots \geqslant \lambda_{j}^{p}$. Матрица $\Lambda_{j}$ называется матрицей нормирований, а ее элементы называются нормированиями системы в точке $a_{j}$. Числа 
$\beta_{j}^{k}=\rho_{j}^{k}+\lambda_{j}^{k}$ называются показателями фуксовой системы в точке $a_{j}$. Множество упорядоченных целочисленных наборов $\Lambda_{j}=\left(\lambda_{j}^{1}, \ldots, \lambda_{j}^{p}\right), j=1, \ldots, n$, с невозрастающими элементами $\lambda_{j}^{1} \geqslant \cdots \geqslant \lambda_{j}^{p}$ называется допустимым набором нормирований.

Теперь сформулируем проблему Римана-Гильберта в классе фуксовых систем. Пусть задано множество точек $A=\left\{a_{1}, \ldots, a_{n}\right\}$ на сфере Римана, и пусть задано представление

$$
\chi: \pi_{1}\left(X, z_{0}\right) \rightarrow \mathrm{GL}(p, \mathbb{C}),
$$

где $X=\overline{\mathbb{C}} \backslash A \subset \overline{\mathbb{C}}$. Требуется найти фуксову систему вида $(2)$ или $\left(2^{\prime}\right)$ на сфере Римана $\overline{\mathbb{C}}$, представление монодромии $\chi_{m}$ которой, относительно некоторой фундаментальной матрицы решений $Y(z)$, совпадает с представлением $\chi$, т. е. $\chi(\gamma)=\chi_{m}(\gamma)$ для любого элемента $\gamma \in \pi_{1}\left(X, z_{0}\right)$. Отметим, что мы получим эквивалентную формулировку проблемы Римана-Гильберта, если для некоторой системы образующих $\gamma_{j}, j=1, \ldots n, \prod_{j=1}^{n} \gamma_{j}=1$, в $\pi_{1}\left(X, z_{0}\right)$ будем требовать совпадения матриц $\chi\left(\gamma_{j}\right)$ и матриц монодромии $G_{j}=\chi_{m}\left(\gamma_{j}\right)$ относительно некоторой фундаментальной матрицы решений $Y(z)$, искомой фуксовой системы.

Имеется очень близкий к фуксовым системам класс систем уравнений, называемых регулярными системами. Регулярная система на сфере Римана с множеством особенностей $A=\left\{a_{1}, \ldots, a_{n}\right\}$ - это линейная система мероморфных дифференциальных уравнений

$$
\frac{\mathrm{d} y(z)}{\mathrm{d} z}=B(z) y(z)
$$

где $B(z)$ - мероморфная матрица размера $p \times p$, имеющая полюсы в точках множества $A$, причем решения $y(z)=\left(y_{1}(z), \ldots, y_{p}(z)\right)^{t}$ в любом секторе конечного раствора с вершиной в любой особой точке $a_{j}$ из $A$ имеют не более чем степенной рост. Последнее означает, что при стремлении переменной $z$ по выбранному сектору к его вершине $a_{j}$ выполняется неравенство $\|y(z)\|=$ $\sqrt{\left|y_{1}(z)\right|^{2}+\cdots+\left|y_{p}(z)\right|^{2}} \leqslant$ const $\cdot\left|z-a_{j}\right|^{-N}$ для некоторого целого числа $N$. Для регулярной системы (в общем случае для произвольной мероморфной системы) представление монодромии определяется точно так же, как и для фуксовой системы.

Все фуксовы системы являются регулярными [2], [16], [19]. Для регулярной системы в окрестности любой особой точки имеет место разложение вида (4), в котором матрица $U_{j}(z)$ - голоморфна, но не обязательно голоморфно обратима. Голоморфная обратимость $U_{j}(z)$ в окрестности особой точки является локальным критерием фуксовости (т. е. в рассматриваемой точке $a_{j}$ у матрицы системы $B(z)$ должен быть полюс первого порядка) в этой особой точке. Из сказанного выше ясно, что для регулярных систем определены нормирования в особых точках и матрицы нормирований, а также показатели в особых точках. Для регулярных систем сумма $\Sigma$ всех показателей по всем особым точкам является целым неположительным числом, $\Sigma \leqslant 0$. Равенство нулю суммы показателей регулярной системы, $\Sigma=0$, является глобальным критерием 
фуксовости регулярной системы на сфере Римана. Оба критерия фуксовости, сформулированные выше, принадлежат Левелю [9].

Можно сформулировать теперь ослабленный вариант проблемы РиманаГильберта, всюду заменив в ее формулировке слова "фуксова система" на слова "регулярная система".

Ослабленный вариант проблемы Римана-Гильберта, согласно теореме Племеля [2], [10], [16], [19], [20] всегда имеет положительное решение. Т. е. любое представление $\chi: \pi_{1}\left(X, z_{0}\right) \rightarrow \mathrm{GL}(p, \mathbb{C})$ фундаментальной группы проколотой сферы Римана $X=\overline{\mathbb{C}} \backslash A \subset \overline{\mathbb{C}}$ реализуется как представление монодромии регулярной системы с множеством особых точек $A=\left\{a_{1}, \ldots, a_{n}\right\}$.

Конструкция Болибруха первого контрпримера к проблеме Римана-Гильберта в классе фуксовых систем показывает, что класс регулярных систем шире, чем класс фуксовых систем. Им была предъявлена регулярная система трех уравнений с четырьмя особыми точками, представление монодромии которой не реализуется как представление монодромии фуксовой системы [19], [21]. Точнее, мероморфными калибровочными преобразованиями заданную регулярную систему нельзя привести к фуксовой системе. Эти результаты А. А. были досконально проверены Д.В. Аносовым и по мотивам такой проверки были изложены в усовершенствованной форме в работах [14], [15].

Напомним, что при калибровочных преобразованиях фундаментальной матрицы решений $\widetilde{Y}(z)=M(z) Y(z)$ матрица системы $B(z)$ преобразуется по формуле

$$
\widetilde{B}(z)=\frac{\mathrm{d} M(z)}{\mathrm{d} z} M^{-1}(z)+M(z) B(z) M^{-1}(z) .
$$

Отметим, что калибровочно эквивалентные системы имеют одно и то же представление монодромии, точнее, калибровочные замены фундаментальной матрицы решений не меняют представления монодромии. Сформулированное утверждение о контрпримере означает, что для $p=3$ и $n=4$ множество классов мероморфной калибровочной эквивалентности фуксовых систем с заданным множеством особенностей является собственным подмножеством множества классов мероморфной калибровочной эквивалентности регулярных систем с таким же множеством особенностей. Множество классов мероморфной калибровочной эквивалентности еще называют пространством модулей соответственно фуксовых или регулярных систем. Для $p=2$ и любого $n \geqslant 2$ из результата У. Деккерса [22] следует, что в этом случае множества классов мероморфной калибровочной эквивалентности регулярных и фуксовых систем совпадают. В общем случае предположение о разрешимости проблемы Римана-Гильберта в классе фуксовых систем для представления монодромии регулярной системы влечет калибровочную эквивалентность последней фуксовой системе. Действительно, рассмотрим регулярную систему и ее представление монодромии. В силу предположения о разрешимости проблемы Римана-Гильберта для представления монодромии этой регулярной системы можно взять фуксову систему с теми же особыми точками и матрицами монодромии, что и у исходной фуксовой системы, т.е. в окрестности неособой точки $z_{0}$ наших систем выберем фундаментальные матрицы решений $Y_{1}(z)$ и $Y_{2}(z)$ соответственно для регулярной и фуксовой систем с одинаковыми матрицами монодромии в каждой особой точке. Матричная функция $M(z)=Y_{1}(z) Y_{2}^{-1}(z)$ является однозначной 
функцией на сфере Римана и имеет степенной рост в особых точках. Следовательно, $M(z)$ является мероморфной функцией на сфере Римана, голоморфно обратимой вне заданного множества особых точек, а потому даже рациональной функций, т. е. все ее элементы равны отношениям многочленов. Замена $Y_{1}(z)=M(z) Y_{2}(z)$ осуществляет калибровочную эквивалентность исходной регулярной системы и фуксовой системы с такой же монодромией. В работе А. А. [23], а также в [16] показано, что для любых размерностей представлений $p \geqslant 3$ и любого количества особых точек $n \geqslant 4$, а также для значений этих параметров $p=4$ и $n=3$ существуют серии контрпримеров к проблеме Римана-Гильберта. В работе ученика Андрея Андреевича И. В. Вьюгина [24] для $p \geqslant 3$ и $n \geqslant 4$ построены другие, в чем-то более простые, серии контрпримеров к проблеме Римана-Гильберта. С другой стороны, в силу теоремы Племеля любое представление $\chi: \pi_{1}\left(X, z_{0}\right) \rightarrow \mathrm{GL}(p, \mathbb{C})$ реализуется как представление монодромии регулярной системы. Из последних двух сформулированных утверждений следует, что во всех указанных случаях множество классов мероморфной калибровочной эквивалентности фуксовых систем является собственным подмножеством множества классов мероморфной калибровочной эквивалентности регулярных систем. Для $p=3$ и $n=3$ А. А. доказал [23], что проблема Римана-Гильберта всегда разрешима. Итак, как объяснено выше, только в размерности два и для представлений размерности три с тремя особыми точками имеем совпадение множеств классов калибровочной эквивалентности фуксовых и регулярных систем, что в некотором смысле, в специальных случаях, обобщает на системы мероморфных дифференциальных уравнений хорошо известный результат для скалярных мероморфных дифференциальных уравнений о совпадении множества фуксовых уравнений заданного порядка (в частности, и для порядка два и три) с множеством регулярных уравнений такого же порядка. В итоге получаем, что представление монодромии системы является важнейшим параметром системы, позволяющим выявлять тонкие различия между различными подмножествами в множестве мероморфных линейных систем дифференциальных уравнений.

\section{3. Приводимость и неприводимость представлений и систем дифференциальных уравнений}

Теперь обратимся к описанию различных вариантов понятий неприводимости и приводимости представлений и систем, роль которых в теории линейных мероморфных систем впервые была замечена и активно использована А. А. Болибрухом. Уже в первых публикациях А. А. [19], [21] по классической проблеме Римана-Гильберта, в которых анонсировалось и доказывалось существование контрпримера к разрешимости этой проблемы в классе фуксовых систем, была отмечена роль неприводимости представления монодромии для положительной разрешимости проблемы Римана-Гильберта. В [19], [21] утверждалось, что любое неприводимое представление фундаментальной группы $\Gamma=\pi_{1}\left(X, z_{0}\right)$ в $\mathrm{GL}(3, \mathbb{C})$ реализуется как представление монодромии фуксовой системы трех уравнений. Первый контрпример к проблеме Римана-Гильберта был построен для представления группы $\Gamma$ в $\mathrm{GL}(3, \mathbb{C})$, которое определялось как представление монодромии регулярной (но не фуксовой) системы. Результат Деккерса 
1979 г. [22], упомянутый выше, влечет, что любое двумерное представление группы Г реализуется как представление монодромии фуксовой системы. Поэтому контрпример был возможен, начиная с представлений и систем размерности три, где он и был построен $(p=3, n=4)$. Правда, он оказался самым чутким, из ныне известных, к изменению положения особых точек. В бо́льших размерностях имеются более простые и грубые примеры, когда представление группы $\Gamma$ не реализуется как представление монодромии фуксовой системы при любом положении особых точек на сфере Римана. Набор разнообразных контрпримеров такого типа был найден в работах [2], [16], [19], [25], [26] среди представлений из нового класса представлений, впервые введенного в рассмотрение Болибрухом. Представления из нового класса были позже названы Ю. С. Ильяшенко (см. [27; с. 35]) Б-представлениями в честь А.А. Болибруха. Б-представления - это класс приводимых представлений, для каждого представления $\chi$ из которого матрицы $\chi\left(\gamma_{i}\right)=G_{i}, i=1, \ldots, n$, определяющие представление $\chi$, в своей жордановой нормальной форме имеют только одну жорданову клетку. Регулярные или фуксовы системы, представления монодромии которых являются Б-представлениями, называют Б-системами. Чуть позже в работе [28] А. А. доказал общий результат (одновременно с В. П. Костовым [29], подробный текст доказательства см. в [30]), что любое неприводимое линейное представление группы $Г$ в группу $\mathrm{GL}(p, \mathbb{C})$ можно реализовать как представление монодромии фуксовой системы на сфере Римана. Однако, если фиксировать не только особые точки и матрицы неприводимого представления монодромии в особых точках, но и нормирования искомой фуксовой системы в особых точках, то в этом случае проблема Римана-Гильберта не всегда разрешима. А. А. привел соответствующий пример [16], [19]. Наборы нормирований, для которых проблема Римана-Гильберта имеет отрицательное решение, были названы запрещенными наборами нормирований. Возникло предположение, что для неприводимых представлений число запрещенных наборов нормирований есть всегда конечное число. В статье [31] А. А. привел пример фуксовой системы второго порядка $p=2$ с пятью особыми точками и неприводимой монодромией и доказал, что для ее представления монодромии существует бесконечное число наборов запрещенных нормирований.

Из результата о реализуемости неприводимых представлений как представлений монодромии фуксовых систем, в частности, следует, что выбранное в первом контрпримере А. А. представление монодромии регулярной системы является приводимым. Приводимость представления монодромии явно заданной регулярной системы $\frac{\mathrm{d} y(z)}{\mathrm{d} z}=B(z) y(z)$ из контрпримера в [21] можно также вывести из того, что матрица системы $B(z)$ является матрицей с “нулевым углом", что влечет существование фундаментальной матрицы решений этой системы с таким же "нулевым углом" и, как следствие этого, наличие у всех матриц монодромии такого же (одинакового для всех матриц) "нулевого угла". Последнее означает приводимость представления монодромии. Это наблюдение о связи приводимости системы и приводимости монодромии привело к самостоятельному изучению понятия приводимости систем (полезное и часто используемое средство во многих задачах в теории систем линейных дифференциальных уравнений) и постановке новых задач в аналитической теории дифференци- 
альных уравнений. А.А. получил ряд результатов о разрешимости проблемы Римана-Гильберта для подпредставлений и факторпредставлений представления монодромии регулярной или фуксовой системы и о связи структуры приводимости представления монодромии со структурой приводимости самой системы. Далее А.А. был поставлен вопрос: как структура приводимости представления может влиять на разрешимость проблемы Римана-Гильберта? В частности, возникла задача: описать такие приводимые представления, которые можно реализовать как представления монодромии фуксовой системы с той же структурой приводимости, что и представление.

Теперь напомним, более детально, сведения о приводимости представлений монодромии и систем дифференциальных уравнений и изложим основные результаты, полученные А. А. и его учениками в этом направлении.

Представление монодромии

$$
\chi_{m}: \pi_{1}\left(X, z_{0}\right) \rightarrow \mathrm{GL}(p, \mathbb{C})
$$

мероморфной системы

$$
\frac{\mathrm{d} y(z)}{\mathrm{d} z}=B(z) y(z)
$$

называется приводимым, если оно имеет инвариантное подпространство размерности $l<p$ в пространстве решений этой системы.

В случае приводимого представления монодромии системы (7) в пространстве решений этой системы можно выбрать базис $\mathbf{e}=\left(e_{1}, \ldots, e_{p}\right)$, первые $l$ элементов которого задают базис в инвариантном подпространстве. Возьмем фундаментальную матрицу решений $Y(z)$ системы (7), отвечающую базису е $\left(i\right.$-м столбцом матрицы $Y(z)$ является $i$-й элемент $e_{i}$ базиса е). Тогда любая матрица монодромии $G_{\gamma}=\chi_{m}(\gamma), \gamma \in \pi_{1}\left(X, z_{0}\right)$ относительно фундаментальной матрицы решений $Y(z)$ (напомним, что $Y(z)=\tilde{Y}(z) G_{\gamma}, \tilde{Y}(z)$ - это аналитическое продолжение $Y(z)$ вдоль петли $\gamma$ ) имеет "нулевой угол"

$$
G_{\gamma}=\left(\begin{array}{cc}
G_{\gamma}^{1} & \star \\
0 & G_{\gamma}^{2}
\end{array}\right)
$$

Матрицы $G_{\gamma}^{1}$ имеют размер $l \times l$ и задают представление фундаментальной группы $\pi_{1}\left(X, z_{0}\right)$ в полную линейную группу $\mathrm{GL}(l, \mathbb{C})$, которое определяется действием фундаментальной группы $\pi_{1}\left(X, z_{0}\right)$ на инвариантном подпространстве. Матрицы $G_{\gamma}^{2}$ имеют размер $(p-l) \times(p-l)$ и являются матрицами представления $\pi_{1}\left(X, z_{0}\right)$ в факторпространстве пространства решений по выбранному инвариантному подпространству; эти матрицы могут быть записаны относительно базиса, представленного последними $p-l$ векторами базиса е. Последнее представление называют факторпредставлением, а представление в инвариантном подпространстве называют подпредставлением. При фиксации системы образующих в фундаментальной группе выбором подходящей фундаментальной матрицы решений можно все матрицы монодромии, отвечающие этим образующим, сделать матрицами с одинаковым нулевым углом. Или, другими словами, все матрицы монодромии сопряжением с помощью одной постоянной матрицы можно привести к матрицам с одинаковым нулевым углом. Приводимое представление монодромии называется вполне приводимым (или, 
в другой терминологии, разложимым представлением), если выбором подходящей фундаментальной матрицы решений все матрицы монодромии приводятся к блочно-диагональному виду

$$
G_{\gamma}=\left(\begin{array}{cc}
G_{\gamma}^{1} & 0 \\
0 & G_{\gamma}^{2}
\end{array}\right)
$$

Вся терминология сохраняется и в общей ситуации, когда рассматривается представление произвольной группы в линейном пространстве, но мы преднамеренно объяснили терминологию сначала для представлений монодромии. В случае вполне приводимого представления $\chi$ произвольной группы $Г$ подходящим выбором базиса в пространстве представления все матрицы представления $G_{\gamma}=\chi(\gamma), \gamma \in \Gamma$, можно привести к виду

$$
G_{\gamma}=\left(\begin{array}{ccccc}
G_{\gamma}^{1} & 0 & 0 & \ldots & 0 \\
0 & G_{\gamma}^{2} & 0 & \ldots & 0 \\
\vdots & \vdots & \vdots & \ddots & \vdots \\
0 & 0 & 0 & \ldots & G_{\gamma}^{s}
\end{array}\right)
$$

где для каждого $j=1, \ldots, s$ матрицы $G_{\gamma}^{j}$ задают некоторое неразложимое представление $\chi^{j}$ группы $Г$. В этом случае представление $\chi$ называется прямой суммой представлений $\chi^{j}, j=1, \ldots, s$, и записывается в виде $\chi=\chi^{1} \oplus \cdots \oplus \chi^{s}$.

Приводимость мероморфных систем

$$
\frac{\mathrm{d} y(z)}{\mathrm{d} z}=B(z) y(z)
$$

с заданным множеством особенностей $A=\left\{a_{1}, \ldots, a_{n}\right\} \subset \overline{\mathbb{C}}$ рассматривается относительно мероморфных калибровочных преобразований фундаментальной матрицы решений

$$
\widetilde{Y}(z)=M(z) Y(z)
$$

и матрицы системы

$$
\widetilde{B}(z)=\frac{\mathrm{d} M(z)}{\mathrm{d} z} M^{-1}(z)+M(z) B(z) M^{-1}(z),
$$

где мероморфная матричная функция имеет полюсы в точках из множества $A$ и голоморфно обратима вне этого множества. Если существует мероморфное калибровочное преобразование $M(z)$, приводящее матрицу коэффициентов $B(z)$ системы (8) к матрице

$$
\widetilde{B}(z)=\left(\begin{array}{cc}
B_{1}(z) & \star \\
0 & B_{2}(z)
\end{array}\right)
$$

с нулевым углом, причем матричная функция $B_{1}(z)$ имеет размер $l \times l$ для некоторого $l<p$, то система называется приводимой. Если не существует указанного мероморфного калибровочного преобразования, то система (8) называется неприводимой. 
Только что введенное понятие приводимости системы иногда называют глобальной приводимостъю системы. Мероморфные калибровочные преобразования для приведения системы к биркгофовой стандартной форме осуществляются в окрестности одной особой точки, и их надо рассматривать как локальные калибровочные преобразования. Однако сразу отметим, что результат А. А. [32] об аналитическом приведении к биркгофовой стандартной форме неприводимых мероморфных систем (8) с двумя особыми точками $A=\{z=0$, $z=\infty\}$ на сфере Римана основан на глобальном рассмотрении системы на всей сфере Римана и ее глобальной неприводимости. Подробнее об этом будет сказано в разделе 6.

Для мероморфной системы (8) система с матрицей коэффициентов $B_{1}(z)$, полученная после приведения (8), называется подсистемой системы (8), а мероморфная система с матрицей коэффициентов $B_{2}(z)$ называется факторсистемой системы (8).

Полная приводимость системы (8) (или, в другой терминологии, разложимость системы) означает приводимость матрицы коэффициентов системы $B(z)$ некоторым мероморфным калибровочным преобразованием $M(z)$ к блочно-диагональному виду

$$
\widetilde{B}(z)=\left(\begin{array}{cc}
B_{1}(z) & 0 \\
0 & B_{2}(z)
\end{array}\right)
$$

т. е. система распадается на две независимые системы.

Как показал А. А. [23; лемма 1.3.5] приводимость мероморфной системы влечет такую же приводимость представления монодромии. Полная приводимость представления монодромии для вполне приводимой системы очевидна, так как система распадается на две независимые системы.

Максимальное приведение приводимой системы к ступенчатому виду

$$
B(z)=\left(\begin{array}{ccccc}
B_{1}(z) & \star & \star & \ldots & \star \\
0 & B_{2}(z) & \star & \ldots & \star \\
\vdots & \vdots & \vdots & \ddots & \vdots \\
0 & 0 & 0 & \ldots & B_{s}(z)
\end{array}\right)
$$

означает, что матрицы $B_{j}(z), j=1, \ldots, s$, определяют неприводимые системы. Максимальная приводимость системы влечет приводимость представления монодромии с такой же структурой приведения, но это приведение может быть не максимальным приведением представления монодромии.

Понятие максимального приведения распространяется и на представления монодромии, необходимо лишь в определении заменить матрицы $B(z), B_{j}(z)$, $j=1, \ldots, s$, соответственно на матрицы $G_{\gamma}, G_{\gamma}^{j}, j=1, \ldots, s$.

В классе регулярных систем утверждения, обратные к сформулированным выше утверждениям о типе приводимости представления монодромии для приводимой системы, всегда являются верными. В [23; теорема 5.1.1] А. А. доказал следующую теорему: если представление монодромии $\chi_{m}$ регулярной системы $\frac{\mathrm{d} y(z)}{\mathrm{d} z}=B(z) y(z)$ с множеством особенностей $A=\left\{a_{1}, \ldots, a_{n}\right\}$ приводимо, $\chi_{m}(\gamma)=\left(\begin{array}{cc}G_{\gamma}^{1} & \star \\ 0 & G_{\gamma}^{2}\end{array}\right)$, с матрицей $G_{\gamma}^{1}$ размера $l \times l, l<p$, то эта регулярная 
система также приводима и матрица калибровочно эквивалентной регулярной системы $\widetilde{B}(z)$ имеет вид $\left(\begin{array}{cc}B_{1}(z) & \star \\ 0 & B_{2}(z)\end{array}\right)$, где матрица $B_{1}(z)$ также размера $l \times l$ и имеет полюсы первого порядка во всех точках из $A$, кроме, быть может, одной точки, скажем, $a_{1}$.

Как следствие этой теоремы получается следующий факт: пусть представление монодромии регулярной системы имеет максимальное приведение

$$
G_{\gamma}=\left(\begin{array}{ccccc}
G_{\gamma}^{1} & \star & \star & \ldots & \star \\
0 & G_{\gamma}^{2} & \star & \ldots & \star \\
\vdots & \vdots & \vdots & \ddots & \vdots \\
0 & 0 & 0 & \ldots & G_{\gamma}^{s}
\end{array}\right)
$$

Тогда существует мероморфное калибровочное преобразование $M(z)$, приводящее матрицу регулярной системы к такому же ступенчатому виду

$$
\widetilde{B}(z)=\left(\begin{array}{ccccc}
B_{1}(z) & \star & \star & \ldots & \star \\
0 & B_{2}(z) & \star & \ldots & \star \\
\vdots & \vdots & \vdots & \ddots & \vdots \\
0 & 0 & 0 & \ldots & B_{s}(z)
\end{array}\right)
$$

Подобные утверждения для фуксовых систем в общем случае неверны. В [23; предложение 5.1.1] для $n=4$ и для некоторого расположения четырех точек $\left\{a_{1}, a_{2}, a_{3}, a_{4}\right\}$ на сфере Римана А. А. нашел пример представления фундаментальной группы $\pi_{1}\left(\overline{\mathbb{C}} \backslash\left\{a_{1}, a_{2}, a_{3}, a_{4}\right\}, z_{0}\right)$ в группу обратимых семимерных (т. е. размера $7 \times 7)$ верхнетреугольных матриц, которое реализуется как представление монодромии фуксовой системы, но не реализуется как представление монодромии фуксовой системы с верхнетреугольной матрицей системы. На этом пути А. А. нашел также такое семимерное представление $\pi_{1}\left(\overline{\mathbb{C}} \backslash\left\{a_{1}, a_{2}, a_{3}, a_{4}\right\}, z_{0}\right)$ в группу верхнетреугольных обратимых матриц, которое не реализуется как представление монодромии никакой фуксовой системы. Эти результаты также имеются в книге [16].

Таким образом, проблема Римана-Гильберта имеет отрицательное решение даже для верхнетреугольных представлений. Долгое время считалось, что метод обращения матричных рядов Лаппо-Данилевского может дать положительное решение проблемы Римана-Гильберта для верхнетреугольных представлений. Для частного случая верхнетреугольных представлений - унипотентных представлений, т. е. верхнетреугольных представлений с единицами на главной диагонали, метод обращения дает положительное решение проблемы Римана-Гильберта. Однако из результата А.А. следует, что в общем случае верхнетреугольных представлений обращенные ряды, которые можно выписать более или менее явно, не всегда сходятся.

В работах [16], [23] А. А. были указаны случаи положительного решения задачи реализации приводимых представлений как представлений монодромии приводимых фуксовых систем с той же структурой приводимости системы, что и заданные приводимые представления. А именно, если приводимое представление является представлением монодромии фуксовой системы и сумма всех 
показателей (кроме суммы показателей для особой точки, суммирование показателей также производится и по всем особым точкам), которые соответствуют инвариантному подпространству пространства решений, равна нулю, то эта фуксова система также приводима, и калибровочное приведение системы к виду приводимости монодромии можно осуществить с помощью невырожденной постоянной матрицы. Аналогично, если приводимое представление монодромии фуксовой системы является Б-представлением, т. е. жорданова нормальная форма всех матриц монодромии $G_{j}, j=1, \ldots, n$, состоит из одной клетки, то фуксова система приводима с той же структурой приводимости, что и монодромия, и калибровочное приведение можно осуществить с помощью постоянной невырожденной матрицы. Было также доказано [28], что любое представление фундаментальной группы $\pi_{1}\left(X, z_{0}\right)$ является подпредставлением приводимого представления, которое, в свою очередь, является представлением монодромии фуксовой системы. Аналогично, любое представление является факторпредставлением приводимого представления монодромии фуксовой системы [28]. Эти утверждения можно доказать на основе аналогичных утверждений для систем: любая регулярная система является подсистемой системы, которая мероморфно эквивалентна фуксовой системе и любая регулярная система является факторсистемой системы, мероморфно эквивалентной фуксовой системе [23].

Эти результаты привели А.А. к постановке ряда вопросов, которые он адресовал своим ученикам:

а) если представление монодромии фуксовой системы приводимо, то что можно сказать о приводимости самой системы и каков характер приводимости?

б) если представление монодромии фуксовой системы вполне приводимо (т. е. разложимо в прямую сумму представлений), то разлагается ли сама система в прямую сумму и реализуются ли прямые слагаемые представления монодромии как представления монодромии фуксовых систем?

Сначала А. И. Гладышев (самый старший ученик А. А.) [33] на вопрос о приводимости фуксовых систем с приводимой монодромией дал положительный ответ для систем и представлений размерности четыре $(p=4)$. При этом структуры приводимости монодромии и системы могут не совпадать. В этой же работе он дал полную классификацию четырехмерных представлений с точки зрения разрешимости для них проблемы Римана-Гильберта. Эта классификация в некотором смысле аналогична той, которую получил А.А. для трехмерных представлений. Гладышев привел примеры четырехмерных представлений, которые не реализуются монодромией фуксовых систем, однако отрицательное решение проблемы Римана-Гильберта для этих представлений не сводится к трехмерным контрпримерам, найденным А. А., т. е. трехмерные контрпримеры не являются подпредставлениями или факторпредставлениями построенных четырехмерных контрпримеров.

Вскоре после работ Гладышева С. Малек [34], [35] (французский ученик А. А.) для систем и представлений любой размерности дал положительный ответ на вопрос о приводимости фуксовой системы с приводимым представлением монодромии. И в общем случае, структуры приводимости представления монодромии и фуксовой системы могут не совпадать. 
Для систем размерности 4 с разложимым представлением монодромии $\chi=$ $\chi_{1} \oplus \chi_{2}$, т. е. $\chi(\gamma)=G_{\gamma}=\left(\begin{array}{cc}G_{\gamma}^{1} & 0 \\ 0 & G_{\gamma}^{2}\end{array}\right), G_{\gamma}^{1}=\chi_{1}(\gamma)$ и $G_{\gamma}^{2}=\chi_{2}(\gamma)$, еще в 1994 г. [36] Гладышев доказал, что фуксова система калибровочно эквивалентна разложимой фуксовой системе

$$
\frac{\mathrm{d} y(z)}{\mathrm{d} z}=\left(\begin{array}{cc}
B_{1}(z) & 0 \\
0 & B_{2}(z)
\end{array}\right) y(z)
$$

и представления $\chi_{1}$ и $\chi_{2}$ являются представлениями монодромии фуксовых подсистем. Для представлений и систем произвольной размерности С. Малек в работе [37] доказал аналогичный результат о разложимости системы с разложимой монодромией при дополнительных ограничениях на представление монодромии рассматриваемой фуксовой системы. А именно, если представление монодромии $\chi$ фуксовой системы $\frac{\mathrm{d} y(z)}{\mathrm{d} z}=B(z) y(z)$ вполне приводимо, $\chi=\chi_{1} \oplus \chi_{2}$, и представление $\chi_{1}$ неприводимо, а представление $\chi_{2}$ является приводимым со специальным типом приводимости, что влечет разрешимость для него проблемы Римана-Гильберта, то исходная фуксова система калибровочно эквивалентна разложимой фуксовой системе $\frac{\mathrm{d} y(z)}{\mathrm{d} z}=\left(\begin{array}{cc}B_{1}(z) & 0 \\ 0 & B_{2}(z)\end{array}\right) y(z)$, причем представления монодромии систем $\frac{\mathrm{d} y(z)}{\mathrm{d} z}=B_{i}(z) y(z), i=1,2$, соответственно эквивалентны представлениям $\chi_{i}, i=1,2$.

Без дополнительных ограничений на представление монодромии фуксовой системы ответ на вопрос о разложимости фуксовой системы с разложимой монодромией отрицателен. В 2004 г. И.В.Вьюгин построил соответствующий контрпример [38]. Он рассмотрел (найденную Болибрухом [31]) регулярную систему размерности четыре с пятью особыми точками

$$
\begin{aligned}
& \frac{\mathrm{d} y(z)}{\mathrm{d} z}=\left(\left(\begin{array}{cccc}
3 / 2 & 0 & 0 & 0 \\
0 & 0 & 0 & 1 / z \\
0 & 0 & 1 / 2 & 0 \\
0 & 0 & 0 & 1
\end{array}\right) \frac{1}{z}+\left(\begin{array}{cccc}
-3 / 8 & -3 / 16 & -1 / 4 & 1 / 4 \\
3 / 16 & 0 & 1 / 4 & 0 \\
0 & 0 & -1 / 8 & -1 / 16 \\
0 & 0 & 1 / 16 & -1 / 4
\end{array}\right) \frac{1}{z+1}\right. \\
& +\left(\begin{array}{cccc}
-3 / 8 & -3 / 16 & -5 / 4 & -1 / 4 \\
3 / 16 & 0 & -1 / 4 & 0 \\
0 & 0 & -1 / 8 & -1 / 16 \\
0 & 0 & 1 / 16 & -1 / 4
\end{array}\right) \frac{1}{z-1}+\left(\begin{array}{cccc}
-3 / 8 & 3 / 16 & 3 / 4 & 0 \\
-3 / 16 & 0 & 0 & 1 / 2 \\
0 & 0 & -1 / 8 & 1 / 16 \\
0 & 0 & -1 / 16 & -1 / 4
\end{array}\right) \frac{1}{z+i} \\
& \left.+\left(\begin{array}{cccc}
-3 / 8 & 3 / 16 & 3 / 4 & 0 \\
-3 / 16 & 0 & 0 & -1 / 2 \\
0 & 0 & -1 / 8 & 1 / 16 \\
0 & 0 & -1 / 16 & -1 / 4
\end{array}\right) \frac{1}{z-i}\right) y(z)
\end{aligned}
$$

для которой лишь одна особая точка $z=0$ не фуксова. Эту систему, как показал Болибрух [15], нельзя калибровочными преобразованиями привести к фуксовой системе с теми же особыми точками, т. е. ее представление монодромии $\chi_{2}$ дает контрпример к проблеме Римана-Гильберта. Вьюгин взял прямую сумму $\chi=\chi_{1} \oplus \chi_{2}$ представления $\chi_{2}$ и двумерного представления $\chi_{1}$, 
заданного матрицами

$$
\begin{array}{rlrl}
G_{1}^{0} & =\left(\begin{array}{ll}
1 & 1 \\
0 & 1
\end{array}\right), & G_{1}^{1}=\left(\begin{array}{cc}
1 & 0 \\
-1 & 1
\end{array}\right), \\
G_{1}^{-1} & =\left(\begin{array}{cc}
1 & -1 \\
1 & 0
\end{array}\right), & G_{1}^{i} & =G_{1}^{-i}\left(\begin{array}{ll}
1 & 0 \\
0 & 1
\end{array}\right) .
\end{array}
$$

Верхние индексы выписанных матриц указывают, на какой образующей $\gamma_{a}$ фундаментальной группы $\pi_{1}\left(\overline{\mathbb{C}} \backslash\{0,-1,1,-i, i\}, z_{0}\right)$ рассматривается значение представления $\chi_{1}\left(\gamma_{a}\right)$, при условии, что каждая образующая $\gamma_{a}$ представлена "малой петлей", обходящей точку $a \in\{0,-1,1,-i, i\}$. Для полученного шестимерного представления Вьюгин доказал, что оно реализуется как представление монодромии фуксовой системы. Полученную фуксову систему нельзя разложить с помощью калибровочного преобразования в прямую сумму фуксовых систем с заданными представлениями монодромии $\chi_{1}, \chi_{2}$. В противном случае четырехмерная регулярная система с монодромией $\chi_{2}$ была бы калибровочно эквивалентна фуксовой системе, а это противоречило бы сделанному выбору. Вьюгин также доказал [24], что любое представление можно представить как прямое слагаемое представления монодромии фуксовой системы. Если понимать стабилизацию представления фундаментальной группы как добавление прямого слагаемого к этому представлению, а представления монодромии фуксовых систем называть фуксовыми представлениями, то результат Вьюгина можно сформулировать как утверждение о том, что любое представление фундаментальной группы $\pi_{1}\left(\overline{\mathbb{C}} \backslash A, z_{0}\right)$ является стабильно фуксовым представлением. Причем стабилизирующее представление, с которым мы берем прямую сумму исходного представления, чтобы получить фуксово представление, можно выбрать неприводимым представлением [24]. В работе [24] Вьюгин указал новые достаточные условия для положительной разрешимости задачи Болибруха о разложимости фуксовых систем с разложимой монодромией. Пусть, как и раньше, $\gamma_{1}, \ldots, \gamma_{n}$ - образующие фундаментальной группы $\pi_{1}\left(\overline{\mathbb{C}} \backslash A, z_{0}\right), A=\left\{a_{1}, \ldots, a_{n}\right\}$, представленные “малыми петлями", обходящими соответственно точки $a_{1}, \ldots, a_{n}$, и пусть заданы такие два представления $\chi_{1}$ и $\chi_{2}$, что их прямая сумма $\chi=\chi_{1} \oplus \chi_{2}$ является фуксовым представлением. Показано, что если спектры операторов $\chi_{1}\left(\gamma_{j}\right)$ и $\chi_{2}\left(\gamma_{j}\right)$ не пересекаются для каждого $j=1, \ldots, n$, то фуксова система с представлением монодромии $\chi=\chi_{1} \oplus \chi_{2}$ разложима в прямую сумму фуксовых систем,

$$
\frac{\mathrm{d} y(z)}{\mathrm{d} z}=\left(\begin{array}{cc}
B_{1}(z) & 0 \\
0 & B_{2}(z)
\end{array}\right) y(z)
$$

причем фуксовы системы

$$
\frac{\mathrm{d} y_{1}(z)}{\mathrm{d} z}=B_{1}(z) y_{1}(z) \quad \text { и } \quad \frac{\mathrm{d} y_{2}(z)}{\mathrm{d} z}=B_{2}(z) y_{2}(z)
$$

имеют представления монодромии $\chi_{1}$ и $\chi_{2}$ соответственно.

В заключение этого раздела опишем результаты, которые развивают и усиливают результаты А.А. по исследованию верхнетреугольных систем и верхнетреугольных представлений. Мы уже отмечали, что А.А. в размерности 
семь построил контрпример к проблеме Римана-Гильберта и построил пример фуксова верхнетреугольного представления, которое не реализуется верхнетреугольной фуксовой системой [16], [23]. Достаточные условия для положительной разрешимости проблемы Римана-Гильберта в общем случае, сформулированные в [16], [23] и в [33], приводят к ее положительной разрешимости для представлений размерности не больше четырех, причем разрешимость имеет место в классе верхнетреугольных фуксовых систем. В работе [39] Ж. Вандамм (французская ученица А.А.) доказала, что любое верхнетреугольное представление размерности пять реализуется верхнетреугольной фуксовой системой. Затем в своей диссертации [40] она нашла пример шестимерного верхнетреугольного фуксова представления, которое не реализуется как представление монодромии шестимерной верхнетреугольной фуксовой системы. Там же она предъявила шестимерное верхнетреугольное представление, которое не может быть представлением монодромии никакой фуксовой системы. На основе критерия положительной разрешимости проблемы Римана-Гильберта в классе фуксовых систем, который основан на понятии $J$-типа матриц монодромии и решении в целых числах некоторой системы алгебраических уравнений, Вандамм предъявила алгоритм проверки реализуемости верхнетреугольного представления как представления монодромии фуксовой системы. В частности, доказательство и компьютерные вычисления, используемые для получения положительного ответа в задаче Римана-Гильберта в классе фуксовых верхнетреугольных систем размерности пять и выявления представлений размерности 6 , для которых ответ, возможно, отрицательный, были впоследствии усовершенствованы М. А. Бутузовым (также ученик А. А.) в работе [41].

\section{4. Фуксовы уравнения и фуксовы системы. Иррегулярные системы. Обобщенная проблема Римана-Гильберта}

Исследование различных задач аналитической теории дифференциальных уравнений всегда приводит к естественному вопросу: а что новые результаты и методы дают в применении к скалярным аналитическим дифференциальным уравнениям на сфере Римана, классическому объекту этой теории? В этом разделе мы расскажем о результатах А. А. и его учеников в этом направлении. А также затронем вопрос о различных обобщениях понятий и задач, связанных с проблемой Римана-Гильберта.

Вопросом о разрешимости проблемы Римана-Гильберта в классе фуксовых дифференциальных уравнений порядка $p$ с $n$ особыми точками А. А. занялся сразу после открытия своего контрпримера [42]. Тот факт, что для значений параметров $p=2$ и $n>3$ не любое представление

$$
\chi: \pi_{1}\left(\overline{\mathbb{C}} \backslash\left\{a_{1}, \ldots, a_{n}\right\}, z_{0}\right) \rightarrow \mathrm{GL}(p, \mathbb{C})
$$

можно реализовать как представление монодромии фуксова уравнения порядка $p$ с множеством особых точек $A=\left\{a_{1}, \ldots, a_{n}\right\}$, возможно, знал уже Риман, так как ему принадлежит идея подсчета числа существенных числовых параметров, которыми определяются фуксовы уравнения или представления. На 
современном языке - это выяснение размерностей пространств фуксовых уравнений и пространств представлений фундаментальной группы. Мы будем говорить просто о размерностях пространств уравнений и пространств представлений. При указанных значениях параметров $p=2$ и $n>3$ размерность пространства модулей фуксовых уравнений порядка два и с числом особых точек большим трех, меньше размерности пространства модулей представлений $\chi$ [16] и потому, по размерностным соображениям, не всякое представление $\chi$ можно реализовать как представление монодромии фуксова уравнения. Если вместо уравнений брать фуксовы системы, то названные размерности совпадают. Риман детально исследовал случай $p=2, n=3$; тогда также размерность пространства уравнений совпадает с размерностью пространства представлений. Он доказал, что в ситуации "общего положения" для представления $\chi$ оно всегда реализуется как представление монодромии гипергеометрического уравнения Гаусса [16]. В работе [42] А. А. предъявил двумерное представление, которое не реализуется как представление монодромии гипергеометрического уравнения Гаусса, а следовательно, не реализуется как представление монодромии любого фуксова уравнения второго порядка, так как все такие уравнения редуцируются к гипергеометрическому уравнению Гаусса ${ }^{1}$. Новые случаи положительной разрешимости проблемы Римана-Гильберта и примеры отрицательного ее решения в классе гипергеометрических уравнений Гаусса для некоторых специальных представлений не так давно рассмотрены в работе ученика Андрея Андреевича В. А. Побережного [43].

Чтобы сформулировать результаты, полученные в [42], напомним основные сведения о фуксовых уравнениях.

Фуксовым уравнением порядка $p$ с множеством особых точек $A=\left\{a_{1}, \ldots, a_{n}\right\}$ называется уравнение

$$
y^{(p)}+b_{1}(z) y^{(p-1)}+\cdots+b_{p}(z) y=0
$$

с мероморфными коэффициентами $b_{k}(z), k=1, \ldots, p$, которые имеют полюсы в точках множества $A$, причем для каждой точки $a_{j} \in A$ коэффициент $b_{k}(z)$ имеет в $a_{j}$ полюс порядка не выше $k$, т. е. $b_{k}(z)=r_{k}(z) /\left(z-a_{j}\right)^{k}$, где $r_{k}(z)$ - голоморфная функция в окрестности $a_{j}$. Здесь $y^{(i)}(z)=\frac{\mathrm{d}^{i} y(z)}{\mathrm{d} z^{i}}-i$-я производная функции $y(z)$.

Понятие представления монодромии фуксова уравнения и показателей уравнения в особых точках определяются аналогично этим понятиям для систем. Только в этом случае элементами пространства решений являются не векторфункции, а обычные скалярные функции. Аналогично ставится и проблема Римана-Гильберта в классе фуксовых уравнений. В этом случае преобразования монодромии действуют в пространстве скалярных функций, которое является пространством решений уравнения (13).

Стандартная замена

$$
y_{i}(z)=y^{(i-1)}(z), \quad i=1,2, \ldots, p
$$

\footnotetext{
${ }^{1}$ Собственно, здесь имеются некоторые тонкости, ибо преобразования, о которых идет речь, вообще говоря, изменяют монодромию. Аккуратное рассмотрение вопроса см. в [3].
} 
сводит уравнение (13) к системе

$$
\frac{\mathrm{d}}{\mathrm{d} z}\left(\begin{array}{c}
y_{1} \\
y_{2} \\
\vdots \\
y_{p}
\end{array}\right)=\left(\begin{array}{cccc}
0 & 1 & \ldots & 0 \\
\vdots & \ddots & \ddots & \vdots \\
0 & \cdots & 0 & 1 \\
-b_{p} & \cdots & -b_{2} & -b_{1}
\end{array}\right)\left(\begin{array}{c}
y_{1} \\
y_{2} \\
\vdots \\
y_{p}
\end{array}\right)
$$

Система (14), очевидно, является регулярной системой, а не фуксовой. Кроме особых точек из множества $A=\left\{a_{1}, \ldots, a_{n}\right\}$ на сфере Римана $\overline{\mathbb{C}}$, система (14) имеет, в общем случае, особенность в бесконечно удаленной точке $z=\infty$. А. А. доказал [42], что мероморфными калибровочными преобразованиями с множеством особенностей $A \cup\{\infty\}$ систему (14) можно преобразовать к фуксовой системе с особенностями только в $A$. Этот факт влечет, что если представление (12) является представлением монодромии фуксова уравнения (13), то оно является представлением монодромии фуксовой системы с теми же особыми точками, т. е. положительная разрешимость проблемы Римана-Гильберта для представления $\chi$ в классе фуксовых уравнений влечет ее положительную разрешимость для $\chi$ в классе фуксовых систем. В работе [44] И.В. Вьюгин упростил доказательство А.А. и уточнил, каков вид фуксовой системы, которая имеет те же особые точки и то же представление монодромии, что и исходное фуксово уравнение. Требуемая фуксова система может быть выбрана с нижнетреугольными матрицами вычетов системы $B_{j}$, $j=1, \ldots, n$, во всех точках $a_{1}, \ldots, a_{n}$, кроме одной, например, $a_{1}$, в которой матрица вычетов $B_{1}$ может иметь ненулевые элементы ниже диагонали и ненулевую наддиагональ, состоящую из единиц.

В начале раздела было отмечено, что, по соображениям размерности, не любое представление фундаментальной группы (12) является представлением фуксова уравнения (13). Соответствующие пространства определяются разным числом параметров. Чтобы уравнять размерность пространства уравнений и размерность пространства представлений, разрешим фуксову уравнению (13) иметь дополнительные особые точки, кроме особых точек из множества $A$. Причем матрицы монодромии, отвечающие дополнительным особым точкам, можно полагать равными единице. Иными словами, мы полагаем, что решения уравнения (13) не имеют ветвления около дополнительных особых точек. Так что группа монодромии, которая есть образ представления монодромии всей фундаментальной группы в группу матриц, не изменяется. Такие дополнительные особые точки называют ложными особыми точками уравнения.

Возникает вопрос: для заданного представления $\chi(12)$, какое минимальное число $m$ ложных особых точек $t_{1}, \ldots, t_{m}$ необходимо добавить, чтобы реализовать представление $\chi$ как представление монодромии фуксова уравнения (13) с $n+m$ особыми точками, причем $m$ - из них ложные особые точки?

В работе [42] для неприводимых представлений $\chi$ (12) А. А. доказал следующую оценку для минимального числа ложных особых точек $m$ :

$$
m \leqslant \frac{p(p-1)}{2}(n-2)-\sum_{i=1}^{p}\left(k_{1}-k_{i}\right)+1-l,
$$


где целые числа $k_{i}, i=1, \ldots, p$, образуют невозрастающую последовательность $k_{1} \geqslant \cdots \geqslant k_{p}$, a $l-$ это число первых равных между собой чисел из набора $k_{1}, \ldots, k_{p}$, т. е. $k_{1}=\cdots=k_{l}$. Целые числа $k_{1}, \ldots, k_{p}$ связаны с представлением $\chi$ следующим образом. По представлению $\chi$, относительно некоторого покрытия кругами дополнения $X=\overline{\mathbb{C}} \backslash\left\{a_{1}, \ldots, a_{n}\right\}$, строится голоморфное векторное расслоение $E$ со слоем $\mathbb{C}^{p}$ на $X$ с постоянными функциями перехода. В этом расслоении имеется интегрируемая связность, определенная обычным дифференциалом голоморфных функций. Для этого расслоения с голоморфной связностью строится каноническое продолжение на сферу Римана $\overline{\mathbb{C}}$ по Делиню [12] и Манину [11]. Получаем голоморфное расслоение $E(0)$ на $\overline{\mathbb{C}}$ с логарифмической связностью. Голоморфное расслоение $E(0)$, согласно теореме Биркгофа-Гротендика, распадается в прямую сумму одномерных голоморфных расслоений

$$
E(0) \simeq \mathscr{O}\left(-k_{1}\right) \oplus \cdots \oplus \mathscr{O}\left(-k_{p}\right),
$$

где $k_{1} \geqslant \cdots \geqslant k_{p}$, а $\mathscr{O}(-q)$ обозначает $q$-ю степень расслоения Хопфа $\mathscr{O}(-1)$. Более подробные сведения об этой конструкции и теореме Биркгофа-Гротендика можно найти в книге А. А. [2; лекции 7-9].

Для неприводимых представлений А. А. получил точное выражение для минимального числа ложных особых точек, введя новое понятие максимального фуксова веса представления. Для определения максимального фуксова веса рассмотрим, кроме канонического продолжения $E(0)$, все продолжения $E(\Lambda)$ расслоения $E$, отвечающие всевозможным наборам допустимых нормирований $\Lambda=\left(\Lambda_{1}, \ldots, \Lambda_{n}\right)$ в особых точках $a_{1}, \ldots, a_{n}$. Напомним, что каждое $\Lambda_{j}=\left(\lambda_{j}^{1} \geqslant\right.$ $\left.\cdots \geqslant \lambda_{j}^{p}\right), j=1, \ldots, n,-$ это невозрастающий набор целых чисел (см. [2]). Согласно теореме Биркгофа-Гротендика, получаем разложения

$$
E(\Lambda) \simeq \mathscr{O}\left(-k_{1}(\Lambda)\right) \oplus \cdots \oplus \mathscr{O}\left(-k_{p}(\Lambda)\right), \quad k_{1}(\Lambda) \geqslant \cdots \geqslant k_{p}(\Lambda)
$$

Упорядоченный набор $\left(k_{1}(\Lambda), \ldots, k_{p}(\Lambda)\right)$ невозрастающих целых чисел называется типом расщепления расслоения $E(\Lambda)$.

Рассмотрим величину

$$
\gamma(E(\Lambda))=\sum_{i=1}^{p}\left(k_{1}(\Lambda)-k_{i}(\Lambda)\right),
$$

которая называется фуксовым весом расслоения $E(\Lambda)$.

По определению, максимальный фуксов вес представления $\chi$ равен

$$
\gamma_{\max }(\chi)=\sup _{\Lambda} \gamma(E(\Lambda))=\sup _{\Lambda} \sum_{i=1}^{p}\left(k_{1}(\Lambda)-k_{i}(\Lambda)\right) .
$$

Точная верхняя грань берется по всем нормированиям $\Lambda$.

Максимальный фуксов вес конечен тогда и только тогда, когда представление $\chi$ неприводимо [16], [23]. Формула для минимального числа ложных особых точек для фуксова уравнения с представлением монодромии, эквивалентным заданному неприводимому представлению $\chi$, имеет вид

$$
m=\frac{(n-2) p(p-1)}{2}-\gamma_{\max }(\chi) .
$$

Подобно максимальному фуксову весу представления определяется минимальный фуксов вес представления [45]. Для любого представления $\chi$ как 
в $(12)$, соответствующего ему расслоения $E(\Lambda)$ и его разложения $(15)$ неотрицательное целое число

$$
\gamma_{\min }(\chi)=\min _{\Lambda}\left(k_{1}(\Lambda)-k_{p}(\Lambda)\right)
$$

называется минимальным фуксовым весом представления $\chi$. В определении минимум берется по всем допустимым нормированиям $\Lambda$.

Для произвольных представлений в работе [45] И. В. Вьюгин и Р. Р. Гонцов (ученики А.А.), используя лемму Делиня (т. е. процесс обратного перехода с помощью мероморфных калибровочных преобразований от произвольной мероморфной системы к системе вида (14), а затем к мероморфному скалярному уравнению) плюс к этому понятие минимального фуксова веса представления $\gamma_{\min }(\chi)$ и верхнюю оценку для него $\gamma_{\min }(\chi) \leqslant(n-1)(p-1)$, а также тот факт, что любое представление можно реализовать как представление монодромии регулярной системы с одной нефуксовой особой точкой, доказали, что можно выбрать требуемую регулярную систему с рангом Пуанкаре (т. е. порядок полюса матрицы системы в этой точке минус один) в нефуксовой особой точке, не превосходящим величины $(n-1)(p-1)$. На основе полученной оценки для ранга Пуанкаре они доказали, что любое представление можно реализовать как представление монодромии фуксова уравнения с числом $m$ ложных особых точек, удовлетворяющим неравенству

$$
m \leqslant 2^{-1}(n+1) p(p-1)+1 .
$$

В работе [46] И. В. Вьюгин описал представления, которые нельзя реализовать как монодромию фуксовых уравнений без дополнительных ложных особых точек. Это Б-представления и разложимые представления с непересекающимися спектрами компонент разложения, когда группа монодромии имеет не менее трех нетривиальных образующих. В частности, отмечается, что для двумерных представлений такие представления исчерпывают все представления, не реализуемые как представления монодромии фуксовых уравнений без дополнительных ложных особых точек. Как уже было отмечено выше, Побережный предъявил явный вид представлений [43], которые не реализуются как представления монодромии гипергеометрических уравнений Гаусса без дополнительных ложных особых точек. В [46] Вьюгин также сформулировал критерий реализации представления как представления монодромии фуксова уравнения без ложных особых точек, который изложен в его статье в этом номере журнала.

Теперь обратимся к другой классической теме аналитической теории дифференциальных уравнений - исследованию иррегулярных систем на сфере Римана. Линейные мероморфные системы на сфере Римана называются иррегулярными, если они не являются регулярными, т. е. их решения в особых точках могут иметь не степенной рост, а экспоненциальный.

Основными задачами в теории иррегулярных систем до недавнего времени были только локальные задачи. Это, в первую очередь, описание поведения фундаментальной матрицы решений иррегулярных систем в особых точках. Здесь были получены формулы разложения их формальных решений в формальные ряды, которые имеют вид, подобный формулам Левеля (4):

$$
Y_{j}(z)=U_{j}(z)\left(z-a_{j}\right)^{E_{j}} e^{Q\left(1 /\left(z-a_{i}\right)\right)},
$$


где $Q\left(1 /\left(z-a_{j}\right)\right)$ - диагональная матрица с многочленами от $1 /\left(z-a_{j}\right)$ (или от $\left.1 / \sqrt[s]{z-a_{j}}\right)$ на диагонали, $s$ - натуральное число, $U_{j}(z)$ - формальный ряд Лорана по степеням $z-a_{j}$ с конечной главной частью, $E_{j}-$ постоянная матрица. Более детальные сведения смотри в обзоре [3]. Второй тип давно исследуемых локальных задач для иррегулярных систем - это задача Биркгофа, о которой речь пойдет в следующем разделе.

Глобальные задачи, связанные с обобщенной проблемой Римана-Гильберта и дифференциальной теорией Галуа, стали рассматриваться сравнительно недавно (см. [3], [47], [48] и ссылки в этих работах). А.А. был инициатором исследования обобщенной проблемы Римана-Гильберта [47], где были получены некоторые достаточные условия положительной разрешимости этой задачи. Однако обобщенная проблема Римана-Гильберта допускает много различных трактовок, даже в выборе начальных данных. Во всех рассмотренных случаях получены некоторые результаты о положительной разрешимости обобщенной проблемы Римана-Гильберта в классе иррегулярных систем. Мы не будем останавливаться на подробном описании этих трактовок задачи и результатов в ее исследовании, а сразу отошлем к недавно опубликованному в УМН обстоятельному обзору [3], один из разделов которого как раз посвящен обобщенной проблеме Римана-Гильберта на сфере Римана. В этом обзоре также рассмотрены некоторые варианты проблемы Римана-Гильберта на компактных римановых поверхностях положительного рода и приведены ссылки, которые дают достаточно полное представление об исследованиях в этом направлении. В нем же предложены списки нерешенных задач в рамках различных трактовок обобщенной проблемы Римана-Гильберта.

\section{5. Биркгофова стандартная форма}

Методы, развитые в работах А.А. для исследования проблемы РиманаГильберта, нашли применение в других задачах аналитической теории дифференциальных уравнений. $\mathrm{K}$ их числу относится хорошо известная задача о приведении линейной мероморфной системы в окрестности ее особой точки к биркгофовой стандартной форме.

Проблема Биркгофа состоит в поиске некоторого канонического вида линейной мероморфной системы дифференциальных уравнений в окрестности ее особой точки. Как правило, в качестве особой точки выбирается бесконечно удаленная точка $z=\infty$ и система в окрестности этой точки записывается в следующем виде:

$$
z \frac{\mathrm{d} y}{\mathrm{~d} z}=B(z) y, \quad B(z)=\sum_{m=-\infty}^{r} B_{m} z^{m} .
$$

Если коэффициент $B_{r}$ отличен от нуля, то число $r$ называется рангом Пуанкаре особой точки $z=\infty$. В проблеме Биркгофа требуется калибровочной заменой $\tilde{y}=\Gamma(z) y$ привести систему (19) к полиномиальному виду

$$
z \frac{\mathrm{d} \tilde{y}}{\mathrm{~d} z}=\widetilde{B}(z) \tilde{y}, \quad \widetilde{B}(z)=\sum_{m=0}^{r^{\prime}} \widetilde{B}_{m} z^{m} .
$$


Если матрица $\Gamma(z)$ голоморфна и голоморфно обратима в окрестности $z=\infty$, то говорят об аналитической эквивалентности систем (19) и (20); если же матрица $\Gamma(z)$ и ее обратная матрица мероморфны в точке $z=\infty$, то говорят о мероморфной эквивалентности этих систем. Если в системе $(20) r^{\prime} \leqslant r$, то систему (20) называют биркгофовой стандартной формой системы (19). Заметим, что при аналитических калибровочных заменах ранг Пуанкаре не изменяется. О драматической истории проблемы Биркгофа можно прочитать в [1], [2], [49].

Понятия неприводимости и приводимости системы в смысле раздела 3 , очевидно, определены и в только что описанном локальном случае.

В работе [32] А. А. рассмотрел случай аналитической приводимости к биркгофовой стандартной форме и доказал, что для неприводимых систем эта задача имеет положительное решение для любых размеров системы $p \geqslant 2$. Это было значительным продвижением в теории биркгофовой стандартной формы, где долгое время после работ Биркгофа в начале ХХ в. исследовали проблему для случаев $p=2,3$.

Вопрос о мероморфном приведении к биркгофовой стандартной форме до сих пор полностью не решен. Имеется много различных достаточных условий для ее разрешимости [49]. Хотя любую систему (19) можно привести мероморфным калибровочным преобразованием к виду (20), но с бо́льшим рангом Пуанкаре.

В приводимом случае А. А. доказал [50], что для любой приводимой системы (19) размера $p=4,5$, имеющей в своей максимальной приведенной форме не более двух неприводимых блоков, существует мероморфное калибровочное преобразование, приводящее систему к биркгофовой стандартной форме (20). Этот результат получил некоторое развитие в работе [45], в которой рассмотрен случай произвольной приводимой системы с неприводимыми блоками размеров $p_{1}, \ldots, p_{m}$ в ситуации максимального приведения. В этом случае систему (19) можно мероморфным калибровочным преобразованием привести к полиномиальному виду (20), где ранг Пуанкаре $r^{\prime}$ допускает оценку сверху $r^{\prime} \leqslant 1+r \max _{1 \leqslant j \leqslant m} p_{j}$. Как следствие этого утверждения получен результат А. Д. Брюно [51]: если исходная система (19) верхнетреугольная, то ее можно мероморфным верхнетреугольным калибровочным преобразованием привести к верхнетреугольной биркгофовой стандартной форме. В работе [52] А. А. начал исследование минимальной биркгофовой стандартной формы.

\section{6. Изомонодромные деформации мероморфных систем. Изомонодромное слияние особых точек}

В этом выпуске журнала содержится обзор Р. Р. Гонцова, В. А. Побережного и Г. Ф. Хельминка о теории изомонодромных деформаций фуксовых и, более общим образом, мероморфных линейных систем. Там говорится о вкладе А. А. в эту теорию и дан обзор последних результатов в данной области, причем подробно разъясняются все нужные понятия. Мы сделаем только несколько замечаний исторического и вводного характера, причем позволим себе не дублировать точных определений, имеющихся в указанном обзоре. Для нас пока 
достаточно намека, что о деформации говорят, когда рассматривается семейство систем, зависящее от некоторых параметров, что в фуксовом случае по традиции такими параметрами являются особые точки системы и что изомонодромность означает сохранение монодромии системы при переходе от одного значения параметров к другому.

$\mathrm{C}$ начала XX в. известны так называемые изомонодромные деформации Шлезингера фуксовых систем.

Создается впечатление, что в прошлом о них говорили таким тоном, будто у фуксовых систем никаких других изомонодромных деформаций нет или по крайней мере другие сводятся к шлезингеровским. То, что это действительно изомонодромные деформации, проверяется просто и давно известно, но другой вопрос - какое положение занимают эти деформации среди всех изомонодромных деформаций. Только около 1980 г. Б. Мальгранж и, с другой стороны, группа японских математиков включили другие изомонодромные деформации (причем не только фуксовых систем) в поле своего зрения. Они более или менее явно указали необходимое и достаточное условие для того, чтобы семейство систем

$$
\frac{\mathrm{d} y(z, a)}{\mathrm{d} z}=B(z, a) y
$$

было изомонодромной деформацией. В нем требуется существование некоторой матричнозначной мероморфной дифференциальной 1-формы $\omega(z, a)$ на пространстве переменных $z, a$. Впрочем, их формулировка не кажется окончательной, а основные научные интересы этих авторов не подразумевали детального исследования подобных вопросов.

А.А. Болибрух [53] получил более совершенную формулировку условий, необходимых и достаточных для того, чтобы семейство систем (21) было изомонодромной деформацией, тоже используя вспомогательную пфаффову систему. Исходя из этого, он получил некоторую классификацию семейств изомонодромных деформаций и выяснил "положение" шлезингеровских семейств в классе всех изомонодромных деформаций фуксовых систем. Оказалось, что в одних случаях к ним действительно все сводится, но в других случаях - нет, хотя все-таки изомонодромные деформации Шлезингера привлекают особое внимание. (Последнее, впрочем, связано не только (не столько?) с их особой ролью как изомонодромных деформаций, но и с их связью с интегрированием некоторых уравнений из "солитонного" круга объектов и с уравнениями Пенлеве.)

На самом деле работы А.А. по изомонодромным деформациям фуксовых систем были выполнены в двух направлениях. Первое направление связано с тем, о чем только что говорилось - с уточнением понятия изомонодромной деформации и четким выделением типов изомонодромных деформаций фуксовых систем, когда параметрами деформации являются их особые точки, - это нормализованные шлезингеровские деформации, ненормализованные шлезингеровские, нешлезингеровские деформации. Полученная информация помогла А. А. [53] установить правильную связь изомонодромных семейств фуксовых систем с регулярными системами Пфаффа на многомерных комплексных многообразиях, при этом уравнения изомонодромной деформации выступали как условия интегрируемости Фробениуса для этих систем. А затем он получил новые типы нешлезингеровских изомонодромных деформаций и их некоторую 
классификацию [50]. (Уточнения понятия изомонодромной деформации фуксовых систем, в развитие понятий, введенных А.А., были сделаны в работе Д. В. Аносова [54].) Примеры не шлезингеровских деформаций можно найти в статье [55].

Второе направление связано с исследованием явных локальных уравнений дивизора подвижных полюсов ( $\Theta$-дивизора Мальгранжа) решений уравнений Шлезингера, которые задают нормализованные шлезингеровские деформации. Было известно, что дивизор подвижных особенностей определяется нулями $\tau$-функции Мивы и Джимбо, но явный вид локальных уравнений или хотя бы алгоритм вычисления этих уравнений по начальным данным отсутствовал. А. А. нашел такой алгоритм вычисления локальных уравнений [2], [56]. На этом пути А.А. для изомонодромных деформаций фуксовых систем порядка два с неприводимой монодромией получил значения порядков полюсов решений соответствующих уравнений Шлезингера [21]. Эти порядки полюсов не превосходят двух. Усиление этих результатов содержатся в работе Гонцова [57], где найден локальный вид в окрестности $\Theta$-дивизора решений уравнений изомонодромных деформаций фуксовых систем любых размеров.

На этом мы окончим вводные замечания о достижениях А. А. собственно по теории изомонодромных деформаций и скажем несколько слов о решении А. А. родственной задачи, идущей от В. И. Арнольда, о поведении фуксовой системы при изомонодромном слиянии особых точек. Изомонодромное слияние это когда особые точки разбиты на группы и точки каждой группы в процессе изменения своего положения сливаются в одну особую точку, общую для всех особых точек рассматриваемой группы. Как показал А. А., при таком слиянии фуксова система может стать лишь регулярной и любая регулярная система может быть получена таким способом [58]. В работе [59] Ю. Бибило нашла обобщение этого результата, доказав, что любую мероморфную систему можно получить как результат изомонодромного слияния особых точек системы с минимальными рангами Пуанкаре во всех особых точках. Конечно, при этом надо правильно определить понятие изомонодромной деформации для произвольных линейных мероморфных систем.

\section{7. Методы решения задач аналитической теории дифференциальных уравнений}

Здесь мы перечислим основные методы, используемые в современной аналитической теории дифференциальных уравнений.

Конечно, еще в начале XX века Племель, а затем Биркгоф использовали преобразования линейных мероморфных систем дифференциальных уравнений, которые мы называли мероморфными калибровочными преобразованиями, и для тех же целей - максимального упрощения рассматриваемых систем. В похожем ключе ими пользовался Риман, преобразуя заданные наборы функций. До сих пор калибровочные преобразования являются одним из основных инструментов аналитической теории дифференциальных уравнений. В рамках рассмотрения калибровочных преобразований мероморфных систем 
дифференциальных уравнений Биркгоф пришел к утверждениям об аналитических матричных функциях, определенных в кольце на комплексной плоскости, которые, будучи переведены на современный язык голоморфных расслоений, означают, что любое голоморфное расслоение на сфере Римана распадается в прямую сумму одномерных голоморфных расслоений, что есть суть теоремы Биркгофа-Гротендика. С середины прошлого века голоморфные расслоения прочно вошли в арсенал аналитической теории дифференциальных уравнений. Линейные мероморфные системы дифференциальных уравнений в контексте голоморфных расслоений превращаются в мероморфные связности, а мероморфная тривиализация голоморфных расслоений, наоборот, превращает связности в линейные мероморфные системы уравнений (на самом деле даже регулярные системы, если связность была локально регулярной системой). Калибровочные преобразования трактуются как переход от одного базиса мероморфных сечений к другому или как переход от одного голоморфного расслоения к другому. Наличие мероморфной тривиализации у голоморфных расслоений на сфере Римана легко следует из теоремы Биркгофа-Гротендика. Для каждого одномерного голоморфного слагаемого из этой теоремы тривиализующее его мероморфное сечение можно предъявить в явном виде. Важный аналитический инвариант регулярных систем ввел Левель - это сумма $\Sigma$ показателей, что в терминах голоморфных расслоений есть просто степень этого расслоения (значение первого класса Черна на фундаментальном цикле базы расслоения). Таким образом, равенство нулю суммы показателей, $\Sigma=0$, есть первый шаг для голоморфной тривиальности расслоения (точнее, пока топологической тривиальности). Такая трактовка глобального критерия фуксовости Левеля вела к активному использованию теории голоморфных расслоений и связностей в аналитической теории дифференциальных уравнений. В частности, размышления на эту тему привели А. А. к введению новых аналитических инвариантов - фуксова веса $\gamma(E)$ голоморфного расслоения $E$ на сфере Римана и максимального фуксова веса представления $\gamma_{\max }(\chi)$. Затем в сферу методов аналитической теории вошли понятия стабильного и полустабильного расслоений. Эти понятия первоначально были введены Мамфордом для нужд изучения пространств модулей голоморфных расслоений на компактных римановых поверхностях, а затем приспособлены Э. Эно для нужд дифференциальных уравнений. Она рассматривала голоморфные расслоения в паре с мероморфными связностями в них - и рассматривала понятия стабильности и полустабильности только относительно подрасслоений, которые инвариантны относительно операторов параллельного переноса рассматриваемой связности в расслоении. В дополнение к калибровочным преобразованиям А. А. многократно искусно пользовался понятиями стабильности и полустабильности пар (расслоение, связность). Например, он доказал теорему об эквивалентности задачи существования стабильной пары с заданным представлением монодромии и задачи о разрешимости проблемы Римана-Гильберта в классе фуксовых систем с неприводимым набором коэффициентов [60]. А затем его ученики Вьюгин и Гонцов большинство результатов последних лет получили с помощью комбинации методов теории голоморфных расслоений со связностями и глобальных или локальных калибровочных преобразований. В частности, Вьюгин [61] построил эффективный алгоритм проверки существования стабильной 
пары с заданным представлением монодромии, и, таким образом, согласно теореме Болибруха, получается эффективный алгоритм проверки разрешимости некоторого варианта проблемы Римана-Гильберта для заданного представления - именно, варианта, в котором дополнительно требуется, чтобы искомая фуксова система имела неприводимый набор коэффициентов. На этом пути, с использованием калибровочных преобразований, А. А. нашел эффективный способ вычисления типа расщепления канонического продолжения $E(0)$ на сферу Римана голоморфного расслоения, построенного по представлению монодромии фуксовой системы [62]. Его ученик А. А. Рябов [63] разработал алгоритм и его программную реализацию на ЭВМ (с использованием одного из стандартных математических пакетов) для вычисления типов расщеплений любых продолжений $E(\Lambda)$ на сферу Римана $\overline{\mathbb{C}}$, построенных по представлению монодромии фуксовой системы и любому допустимому набору нормирований $\Lambda_{j}$, $j=1, \ldots, n$.

Мы завершим этот раздел описанием уточнений неравенства Левеля $\Sigma \leqslant 0$ для суммы показателей регулярной системы, полученных Э. Корелем [64]-[66], Р. Р. Гонцовым [67] и А.А. Болибрухом [18]. Эти неравенства также входят в основной арсенал методов аналитической теории дифференциальных уравнений.

В этом направлении первый результат, после оценки Левеля $\Sigma \leqslant 0$, был получен французским математиком Э. Корелем [64]. Он доказал, что для произвольной регулярной системы сумма показателей $\Sigma$ отделена от нуля суммой рангов Пуанкаре, взятой со знаком минус, и имеется оценка снизу. Более точно, для регулярной системы порядка $p$ с $n$ особыми точками $a_{1}, \ldots, a_{n}$, имеющими ранги Пуанкаре $r_{1}, \ldots, r_{n}$, выполняются неравенства Фукса

$$
-\frac{p(p-1)}{2} \sum_{i=1}^{n} r_{i} \leqslant \Sigma \leqslant-\sum_{i=1}^{n} r_{i} .
$$

Аналитическое доказательство этих неравенств и их обобщение на многомерные фуксовы системы, без привлечения алгебраической теории многочленов и формальных степенных рядов, было найдено А. А. в работе [18]. Гонцов в работе [68], с использованием приемов аналитического доказательства Болибруха, улучшил выписанные оценки. Для систем $\frac{\mathrm{d} y}{\mathrm{~d} z}=B(z) y$ вводятся в рассмотрение нильпотентные матрицы $B_{-r_{i}-1}^{i}$ ведущих членов разложения матрицы коэффициентов

$$
B(z)=\frac{B_{-r_{i}-1}^{i}}{\left(z-a_{i}\right)^{r_{i}+1}}+\cdots+\frac{B_{-1}^{i}}{z-a_{i}}+B_{0}^{i}+\cdots, \quad B_{-r_{i}-1}^{i} \neq 0,
$$

в особых точках $a_{1}, \ldots, a_{n}$. Пусть $l_{i}=\operatorname{rk} B_{-r_{i}-1}^{i}$ и $k_{i}=\operatorname{dim} \operatorname{coker} B_{-r_{i}-1}^{i}-$ соответственно ранг и коранг матрицы $B_{-r_{i}-1}^{i}$. Для регулярных систем доказаны следующие неравенства Фукса:

$$
-\frac{p(p-1)}{2} \sum_{i=1}^{n} r_{i}+\sum_{i=1}^{n} \frac{k_{i}\left(k_{i}-1\right)}{2} \leqslant \Sigma \leqslant-\sum_{i=1}^{n} l_{i} r_{i} .
$$

Отметим одно следствие указанных неравенств. Для регулярных систем в случае, когда ведущие матрицы во всех особых точках имеют ранги равные $p-1$, 
неравенства Фукса приобретают вид соотношения Фукса

$$
\Sigma=-\frac{p(p-1)}{2} \sum_{i=1}^{n} r_{i}
$$

Э. Корель [64], [65] также получил подобный результат, но использовал другие приемы рассуждения при выводе следствия из своих неравенств.

Для иррегулярных систем имеются неравенства Фукса подобного вида, полученные Корелем [65] и Гонцовым [68].

Недавно для регулярных систем Корелем [66] найдены дальнейшие уточнения неравенств Фукса. В них помимо рангов нильпотентных матриц $B_{-r_{i}-1}^{i}$ рассматриваются также ранги их ненулевых степеней $l_{i}^{j}=\operatorname{rk}\left(B_{-r_{i}-1}^{i}\right)^{j}$ и их разности $k_{i}^{j}=l_{i}^{j}-l_{i}^{j+1}$. Тогда новые неравенства Фукса имеют вид

$$
-\frac{p(p-1)}{2} \sum_{i=1}^{n} r_{i}+\sum_{i=1}^{n} \sum_{j \geqslant 0} \frac{k_{i}^{j}\left(k_{i}^{j}-1\right)}{2} \leqslant \Sigma \leqslant-\sum_{i=1}^{n} \sum_{j \geqslant 1} l_{i}^{j} r_{i} .
$$

В работе [67] Гонцов использовал свои уточнения неравенств Фукса для усиления оценок сверху А. А. [69], полученных для порядков нулей многочленов многих переменных вдоль траекторий решений регулярных систем. И в этих задачах неприводимость системы влияет на значение оценки.

\section{Список литературы}

[1] Д. В. Аносов, В.П. Лексин, “Андрей Андреевич Болибрух в жизни и науке (30.01.1950 - 11.11.2003)", УМH, 59:6 (2004), 3-22; англ. пер.: D. V. Anosov, V.P. Leksin, "Andrei Andreevich Bolibrukh in life and science (30 January 1950 11 November 2003)", Russian Math. Surveys, 59:6 (2004), 1009-1028.

[2] А.А. Болибрух, Обратные задачи монодромии в аналитической теории дифференииальных уравнений, МЦНМО, М., 2009, ISBN: 978-5-94057-510-8, 220 с., http://biblio.mccme.ru/node/2154.

[3] Р. Р. Гонцов, В. А. Побережный, "Различные варианты проблемы Римана-Гильберта для линейных дифференциальных уравнений”, УМH, 63:4 (2008), 3-42; англ. пер.: R. R. Gontsov, V.A. Poberezhnyi, "Various versions of the RiemannHilbert problem for linear differential equations", Russian Math. Surveys, 63:4 (2008), 603-639.

[4] В. П. Лексин, "О работах А. А. Болибруха по многомерным регулярным и фуксовым системам", УМH, 59:6 (2004), 151-160; англ. пер.: V. P. Leksin, "A. A. Bolibrukh's works on multidimensional regular and Fuchsian systems", Russian Math. Surveys, 59:6 (2004), 1155-1164.

[5] А. А. Болибрух, "О системах Пфаффа типа Фукса", УМН, 32:2 (1977), 203-204.

[6] А.А. Болибрух, "О фундаментальной матрице системы Пфаффа типа Фукса", Изв. АН СССР. Сер. матем., 41:5 (1977), 1084-1109; англ. пер.: A. A. Bolibruh, "On the fundamental matrix of a Pfaffian system of Fuchsian type", Math. USSR-Izv., 11:5 (1977), 1031-1054.

[7] А.А. Болибрух, О фундаментальной матрище систем Пфаффа типа Фукса, Дис. ... канд. физ.-матем. наук, МГУ, М., 1977, 94 с.

[8] А. А. Болибрух, "Пример неразрешимой проблемы Римана-Гильберта на $\mathbb{C} P^{2}$ ", Геометрические методы в задачах алгебры и анализа, вып. 2, Изд-во ЯрГУ, Ярославль, 1980, 60-64. 
[9] A. H. M. Levelt, "Hypergeometric functions. I", Indag. Math., 23 (1961), 361-401.

[10] H. Röhrl, "Das Riemann-Hilbertsche Problem der Theorie der linearen Differentialgleichungen", Math. Ann. B, 133:1 (1957), 1-25.

[11] Yu. Manin, "Moduli Fuchsiani", Ann. Sc. Norm. Sup. Pisa, 19 (1965), 113-126.

[12] P. Deligne, "Équations différentielles à points singuliers réguliers", Lecture Notes in Math., 163 (1970), 133 pp.

[13] N. M. Katz, "An overview of Deligne's work on Hilbert's twenty-first problem", Mathematical developments arising from Hilbert problems (Northern Illinois Univ., De Kalb, Ill., 1974), Proc. Sympos. Pure Math., 28, Amer. Math. Soc., Providence, RI, 1976, $537-557$.

[14] D. V. Anosov, Hilbert 21st problem (according to Bolibruch), Preprint № 660, Inst. Math. Appl., Univ. Minnesota, Minneapolis, 1990, http://www.ima.umn.edu/ preprints/June90Series/660.pdf.

[15] D. V. Anosov, An introduction to Hilbert's 21-st problem, Preprint № 861, Inst. Math. Appl., Univ. Minnesota, Minneapolis, 1991, http://www.ima.umn.edu/preprints/ Aug91Series/861.pdf.

[16] D. V. Anosov, A. A. Bolibruch, The Riemann-Hilbert problem, Aspects Math., E22, Vieweg, Braunschweig, 1994, ISBN: 3-528-06496-X, 190 pp.

[17] Ф. Р. Гантмахер, Теория матрии, Гостехиздат, М., 1953; 4-е изд., Наука, М., 1988, ISBN: 5-02-013722-7, 549 с.; англ. пер.: F. R. Gantmacher, The theory of matrices, 1, 2, Chelsea Publishing Co., New York, 1959.

[18] А.А. Болибрух, "Неравенство Фукса на компактном кэлеровом многообразии", Докл. РАН, 380:4 (2001), 448-451; англ. пер.: A. A. Bolibrukh, "The Fuchs inequality on a compact Kähler manifold", Dokl. Math., 64:2 (2001), 213-215.

[19] А. А. Болибрух, "Проблема Римана-Гильберта", УМH, 45:2 (1990), 3-47; англ. пер.: A. A. Bolibrukh, "The Riemann-Hilbert problem", Russian Math. Surveys, 45:2 (1990), 1-58.

[20] J. Plemelj, Problems in the sense of Riemann and Klein, Interscience Tracts in Pure and Applied Mathematics, 16, Wiley, New York-London-Sydney, 1964, vii+175 pp.

[21] А. А. Болибрух, "Проблема Римана-Гильберта на комплексной проективной прямой", Матем. заметки, 46:3 (1989), 118-120.

[22] W. Dekkers, "The matrix of a connection having regular singularities on a vector bundle of rank 2 on $\mathbb{F}^{1}(C)$ ", Équations differentielles et systèmes de Pfaff dans le champ complexe (Sém., Inst. Rech. Math. Avancée, Strasbourg, 1975), Lecture Notes in Math., 712, Springer, Berlin, 1979, 33-43.

[23] А. А. Болибрух, “21-я проблема Гильберта для линейных фуксовых систем”, Тр. МИАН, 206, Наука, М., 1994, 3-158; англ. пер.: А. А. Bolibrukh, The 21st Hilbert problem for linear Fuchsian systems, Proc. Steklov Inst. Math., 206, Amer. Math. Soc., Providence, RI, 1995, viii, 145 pp.

[24] И.В. Вьюгин, "Фуксовы системы с вполне приводимой монодромией", Матем. заметки, 85:6 (2009), 817-825; англ. пер.: I. V. V'yugin, "Fuchsian systems with completely reducible monodromy", Math. Notes, 85:6 (2009), 780-786.

[25] D. V. Anosov, A simple counterexample to the Fuchsian version of the Hilbert 21 st problem, Preprint № 124, Max-Planck-Institut für Math., Bonn, 1998, http://www.mpim-bonn.mpg.de/preprints/send?bid=242.

[26] D. V. Anosov, "A simple counterexample to the Fuchsian version of the Hilbert 21st problem and related class of monodromy representations", Ulmer Seminare über Funktionalanalysis und Differentialgleichungen, 3 (1998), 1-16.

[27] Ю. С. Ильяшенко, "Три жемчужины теории линейных дифференциальных уравнений (по работам А.А. Болибруха)", УМH, 59:6 (2004), 73-84; англ. пер.: Yu. S. Il'yashenko, "Three gems in the theory of linear differential equations (in the work of A. A. Bolibrukh)", Russian Math. Surveys, 59:6 (2004), 1079-1091. 
[28] А.А. Болибрух, "О достаточных условиях положительной разрешимости проблемы Римана-Гильберта", Матем. заметки, 51:2 (1992), 9-19; англ. пер.: A.A. Bolibrukh, "On sufficient conditions for the positive solvability of the Riemann-Hilbert problem", Math. Notes, 51:2 (1992), 110-117.

[29] V.P. Kostov, "Fuchsian systems on $\mathbb{C} P^{1}$ and the Riemann-Hilbert problem", C. R. Acad. Sci. Paris Sér. I Math., 315:2 (1992), 143-148.

[30] V.P. Kostov, "Monodromy groups of regular systems on the Riemann sphere", Differential equations and quantum groups, IRMA Lect. Math. Theor. Phys., 9, eds. D. Bertrand et al., Eur. Math. Soc., Zürich, 2007, 209-254.

[31] А. А. Болибрух, "К вопросу о существовании фуксовых систем с данными асимптотиками", Динамические системы и смежные вопросы, Сб. статей. К 60-летию со дня рождения академика Дмитрия Викторовича Аносова, Тр. МИАН, 216, Наука, М., 1997, 32-44; англ. пер.: А. A. Bolibrukh, "On the existence of Fuchsian systems with given asymptotics", Proc. Steklov Inst. Math., 216 (1997), 26-37.

[32] А.А. Болибрух, "Об аналитическом преобразовании к стандартной биркгофовой форме", Докл. РАН, 334:5 (1994), 553-555; англ. пер.: А. A. Bolibrukh, "On an analytic transformation to standard Birkhoff form", Dokl. Math., 49:1 (1994), $150-153$.

[33] A. I. Gladyshev, "On the Riemann-Hilbert problem in dimension 4", J. Dyn. Control Syst., 6:2 (2000), 219-264.

[34] M. Stephane, "On reducible monodromies realized by reducible Fuchsian systems", J. Dyn. Control Syst., 5:4 (1999), 509-522.

[35] S. Malek, "Fuchsian systems with reducible monodromy are meromorphically equivalent to reducible Fuchsian systems", Дифференциальные уравнения и динамические системы, Сб. статей. K 80-летию со дня рождения академика Евгения Фроловича Мищенко, Тр. МИАН, 236, Наука, М., 2002, 481-490; Proc. Steklov Inst. Math., 236 (2002), 468-477.

[36] А. И. Гладышев, “О приводимых фуксовых системах четвертого порядка”, Проблемы математики в физических и технических задачах, МФТИ, М., 1994, 66-80.

[37] S. Malek, "On Fuchsian systems with decomposable monodromy", Монодромия в задачах алгебрачческой геометрии и дифференциальных уравнений, Тр. МИАН, 238, Наука, M., 2002, 196-203; Proc. Steklov Inst. Math., 238 (2002), 186-193.

[38] И.В. Вьюгин, "Неразложимая фуксова система с разложимым представлением монодромии", Матем. заметки, 80:4 (2006), 501-508; англ. пер.: I. V. V'yugin, "An indecomposable Fuchsian system with a decomposable monodromy representation", Math. Notes, 80:4 (2006), 478-484.

[39] J. Vandamme, "Problème de Riemann-Hilbert pour une représentation de monodromie triangulaire supérieure de dimension 5", C. R. Acad. Sci. Paris Sér. I Math., 326:9 (1998), 1069-1072.

[40] J. Vandamme, Problème de Riemann-Hilbert pour une représentation de monodromie triangulaire supérieure, Thèse de doctorat en Sciences Mathématiques, Université de Nice-Sophia Antipolis, UFR Faculté des Sciences, Lab. Mathématiques J. A. Dieudonné, 25 mai 1998, 101 pp.

[41] М. А. Бутузов, "Об условиях разрешимости проблемы Римана-Гильберта в классе верхнетреугольных систем", Сборник научных статей аспирантов и соискателей, 80, КГПИ, Коломна, 2006, 193-198.

[42] А.А. Болибрух, "О построении фуксова уравнения по представлению монодромии", Матем. заметки, 48:5 (1990), 22-34; англ. пер.: А. A. Bolibrukh, "Construction of a Fuchsian equation from a monodromy representation", Math. Notes, 48:5 (1990), 1090-1099. 
[43] В. А. Побережный, "О специальных группах монодромии и проблеме РиманаГильберта для уравнения Римана", Матем. заметки, 77:5 (2005), 753-767; англ. пер.: V. A. Poberezhnyi, "On special monodromy groups and the Riemann-Hilbert problem for the Riemann equation", Math. Notes, 77:5 (2005), 695-707.

[44] И. В. Вьюгин, "Построение фуксовой системы по фуксову уравнению", Вестник КГПИ: Математические и естественные науки, 2:3 (2007), 43-47.

[45] И. В. Вьюгин, Р.Р. Гонцов, "О дополнительных параметрах в обратных задачах монодромии", Матем. сб., 197:12 (2006), 43-64; англ. пер.: I. V. V'yugin, R. R. Gontsov, "Additional parameters in inverse monodromy problems", Sb. Math., 197:12 (2006), 1753-1773.

[46] И. В. Вьюгин, "О 21-й проблеме Гильберта для скалярных фуксовых уравнений”, Докл. РАН, 425:3 (2009), 305-308; англ. пер.: V. I. V'yugin, "Hilbert's 21st problem for scalar Fuchsian equations", Dokl. Math., 79:2 (2009), 203-206.

[47] A. A. Bolibruch, S. Malek, C. Mitschi, "On the generalized Riemann-Hilbert problem with irregular singularities", Expo. Math., 24:3 (2006), 235-272.

[48] R. R. Gontsov, I. V. Vyugin, "Some addition to the generalized Riemann-Hilbert problem", Ann. Fac. Sci. Toulouse Math. (6), 18:3 (2009), 561-576.

[49] В. Бальзер, "Проблема приведения Биркгофа", УМН, 59:6 (2004), 41-54; англ. пер.: W. Balser, "Birkhoff's reduction problem", Russian Math. Surveys, 59:6 (2004), 1047-1059.

[50] А. А. Болибрух, "Мероморфное преобразование к биркгофовой стандартной форме в малых размерностях", Солитоны, геометрия, топология - на перекрест$\kappa a x$, Сб. статей. K 60-летию со дня рождения академика Сергея Петровича Новикова, Тр. МИАН, 225, Наука, М., 1999, 87-95; англ. пер.: А. А. Bolibrukh, "Meromorphic transformation to Birkhoff standard form in small dimensions", Proc. Steklov Inst. Math., 225 (1999), 78-86.

[51] А.Д. Брюно, "Мероморфная приводимость линейной треугольной системы ОДУ", Докл. РАН, 371:5 (2000), 587-590; англ. пер.: А. D. Bruno, "Meromorphic reducibility of a linear triangular system of ordinary differential equations", Dokl. Math., 61:2 (2000), 243-246.

[52] А.А. Болибрух, "Минимальная биркгофова стандартная форма", Дифференциалъные уравнения, 39:6 (2003), 723-730; англ. пер.: A. A. Bolibrukh, "The minimal Birkhoff standard form and the reducibility type", Differ. Equ., 39:6 (2003), 759-766.

[53] A. A. Bolibruch, "On isomonodromic deformations of Fuchsian systems", J. Dyn. Control Syst., 3:4 (1997), 589-604.

[54] D. V. Anosov, "Concerning the definition of isomonodromic deformation of Fuchsian systems", Ulmer Seminare über Funktionalanalysis und Differentialgleichungen, 2 (1997), 1-12.

[55] В.А. Побережный, "Общая линейная задача изомонодромной деформации фуксовых систем", Матем. заметки, 81:4 (2007), 599-613; англ. пер.: V.A. Poberezhnyi, "General linear problem of the isomonodromic deformation of Fuchsian systems", Math. Notes, 81:3-4 (2007), 529-542.

[56] А. А. Болибрух, "О tau-функции уравнения изомонодромных деформаций Шлезингера", Матем. заметки, 74:2 (2003), 184-191; англ. пер.: А. A. Bolibrukh, "On the tau function for the Schlesinger equation of isomonodromic deformations", Math. Notes, 74:2 (2003), 177-184.

[57] Р.Р. Гонцов, "О решениях уравнения Шлезингера в окрестности $\Theta$-дивизора Мальгранжа", Матем. заметки, 83:5 (2008), 779-782; англ. пер.: R. R. Gontsov, "On solutions of the Schlesinger equation in the neigborhood of the Malgrange

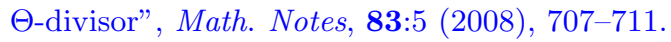

[58] А.А. Болибрух, "Регулярные особые точки как изомонодромные слияния фуксовых", УМН, 56:4 (2001), 135-136; англ. пер.: A. A. Bolibrukh, "Regular singular 
points as isomonodromic confluences of Fuchsian singular points", Russian Math. Surveys, 56:4 (2001), 745-746.

[59] Ю. П. Бибило, "Изомонодромное слияние особых точек", Матем. заметки, 87:3 (2010), 330-336; англ. пер.: Yu. P. Bibilo, "Isomonodromic confluence of singular points", Math. Notes, 87:3-4 (2010), 309-315.

[60] А. А. Болибрух, "Проблема Римана-Гильберта на компактной римановой поверхности", Монодромия в задачах алгебраической геометрии и дифберенииальных уравнений, Тр. МИАН, 238, Наука, М., 2002, 55-69; англ. пер.: A. A. Bolibrukh, "The Riemann-Hilbert problem on a compact Riemann surface", Proc. Steklov Inst. Math., 238 (2002), 47-60.

[61] И.В. Вьюгин, "О конструктивных условиях разрешимости проблемы РиманаГильберта", Матем. заметки, 77:5 (2005), 643-655; англ. пер.: I. V. V'yugin, "Constructive solvability conditions for the Riemann-Hilbert problem", Math. Notes, 77:5 (2005), 595-605.

[62] A. A. Bolibruch, "Vector bundles associated with monodromies and asymptotics of Fuchsian systems", J. Dyn. Control Syst., 1:2 (1995), 229-252.

[63] А.А. Рябов, "Типы расщеплений векторных расслоений, построенных по монодромии заданной фуксовой системы", Журн. вычисл. матем. и матем. физ., 44:4 (2004), 676-685; англ. пер.: A. A. Ryabov, "Splitting types of vector bundles constructed from the monodromy of a given Fuchsian system", Comput. Math. Math. Phys., 44:4 (2004), 640-648.

[64] E. Corel, "Inégalités de Fuchs pour les systèmes différentiels réguliers", C. R. Acad. Sci. Paris Sér. I Math., 328:11 (1999), 983-986.

[65] E. Corel, "Relations de Fuchs pour les systèmes différentiels irréguliers", C. R. Acad. Sci. Paris Sér. I Math., 333:4 (2001), 297-300.

[66] E. Corel, "Exponents of a meromorphic connection on a compact Riemann surface", Pacific J. Math., 242:2 (2009), 259-279.

[67] Р.Р. Гонцов, "О порядках нулей многочлена на траектории решения системы линейных дифференциальных уравнений с регулярными особыми точками", $M a$ тем. заметки, 76:3 (2004), 473-477; англ. пер.: R. R. Gontsov, "Orders of zeros of polynomials on trajectories of solutions of a system of linear differential equations with regular singular points", Math. Notes, 76:3 (2004), 438-442.

[68] Р. Р. Гонцов, "Уточненные неравенства Фукса для систем линейных дифференциальных уравнений”, Изв. РАН. Сер. матем., 68:2 (2004), 39-52; англ. пер.: R. R. Gontsov, "Refined Fuchs inequalities for systems of linear differential equations", Izv. Math., 68:2 (2004), 259-272.

[69] А.А. Болибрух, "Кратности нулей компонент решений системы с регулярными особыми точками", Дифференииальные уравнения и динамические системы, Сб. статей. K 80-летию со дня рождения академика Евгения Фроловича Мищенко, Тр. МИАН, 236, Наука, М., 2002, 61-65; англ. пер.: А. A. Bolibrukh, "Multiplicities of zeros of the components of solutions of a system with regular singular points", Proc. Steklov Inst. Math., 236 (2002), 53-57.

Д. В. Аносов (D. V. Anosov)

Математический институт им. В. А. Стеклова РАН

E-mail: anosov@mi.ras.ru

\section{В. П. Лексин (V.P. Leksin)}

Московский государственный областной социально-гуманитарный институт

E-mail: lexin_vp@mail.ru, lexine@mccme.ru
Поступила в редакцию 02.12 .2010 